\title{
Optimally biosynthesized, PEGylated gold nanoparticles functionalized with quercetin and camptothecin enhance potential anti-inflammatory, anti-cancer and anti-angiogenic activities
}

Priyadarshani S. Sadalage ${ }^{1}$, Reshma V. Patil ${ }^{1 \dagger}$, Darshana V. Havaldar ${ }^{1 \dagger}$, Shruti S. Gavade ${ }^{1}$, Ana Cláudia Santos ${ }^{2,3}$ and Kiran D. Pawar ${ }^{1 *}$

\begin{abstract}
Background: The development of nano delivery systems is rapidly emerging area of nanotechnology applications where nanomaterials (NMs) are employed to deliver therapeutic agents to specific site in a controlled manner. To accomplish this, green synthesis of NMs is widely explored as an eco-friendly method for the development of smart drug delivery system. In the recent times, use of green synthesized NMs, especially metallic NMs have fascinated the scientific community as they are excellent carriers for drugs. This work demonstrates optimized green, biogenic synthesis of gold nanoparticles (AuNPs) for functionalization with quercetin (QT) and camptothecin (CPT) to enhance potential anti-inflammatory, anti-cancer and anti-angiogenic activities of these drugs.

Results: Gold nanoparticles were optimally synthesized in 8 min of reaction at $90^{\circ} \mathrm{C}$, pH 6 , using $4 \mathrm{mM}$ of $\mathrm{HAuCl}_{4}$ and 4:1 ratio of extract: $\mathrm{HAuCl}_{4}$. Among different capping agents tested, capping of AuNPs with polyethylene glycol 9000 (PG9) was found best suited prior to functionalization. PG9 capped AuNPs were optimally functionalized with QT in $1 \mathrm{~h}$ reaction at $70{ }^{\circ} \mathrm{C}, \mathrm{pH}$ 7, using $1200 \mathrm{ppm}$ of QT and 1:4 ratio of AuNPs-PG9:QT whereas, CPT was best functionalized at RT in $1 \mathrm{~h}$, pH 12, AuNPs-PG9:CPT ratio of 1:1, and $0.5 \mathrm{mM}$ of CPT. QT functionalized AuNPs showed good anti-cancer activity $\left(\mathrm{IC}_{50} 687.44 \mathrm{\mu g} / \mathrm{mL}\right)$ against MCF-7 cell line whereas test of anti-inflammatory activity also showed excellent activity $\left(\mathrm{IC}_{50} 287.177 \mathrm{mg} / \mathrm{L}\right)$. The CAM based assessment of anti-angiogenic activity of CPT functionalized AuNPs demonstrated the inhibition of blood vessel branching confirming the anti-angiogenic effect.
\end{abstract}

Conclusions: Thus, present study demonstrates that optimally synthesized biogenic AuNPs are best suited for the functionalization with drugs such as QT and CPT. The functionalization of these drugs with biogenic AuNPs enhances

\footnotetext{
*Correspondence: pawarkiran1912@gmail.com

${ }^{\dagger}$ Reshma V. Patil and Darshana V. Havaldar contributed equally

1 School of Nanoscience and Biotechnology, Shivaji University, Kolhapur,

Maharashtra, India

Full list of author information is available at the end of the article
}

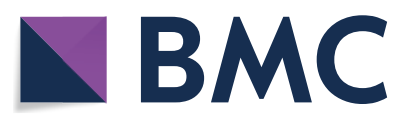

(c) The Author(s) 2021. This article is licensed under a Creative Commons Attribution 4.0 International License, which permits use, sharing, adaptation, distribution and reproduction in any medium or format, as long as you give appropriate credit to the original author(s) and the source, provide a link to the Creative Commons licence, and indicate if changes were made. The images or other third party material in this article are included in the article's Creative Commons licence, unless indicated otherwise in a credit line to the material. If material is not included in the article's Creative Commons licence and your intended use is not permitted by statutory regulation or exceeds the permitted use, you will need to obtain permission directly from the copyright holder. To view a copy of this licence, visit http://creativeco mmons.org/licenses/by/4.0/. The Creative Commons Public Domain Dedication waiver (http://creativecommons.org/publicdomain/ zero/1.0/) applies to the data made available in this article, unless otherwise stated in a credit line to the data. 
the potential anti-inflammatory, anti-cancer and anti-angiogenic activities of these drugs, therefore can be used in
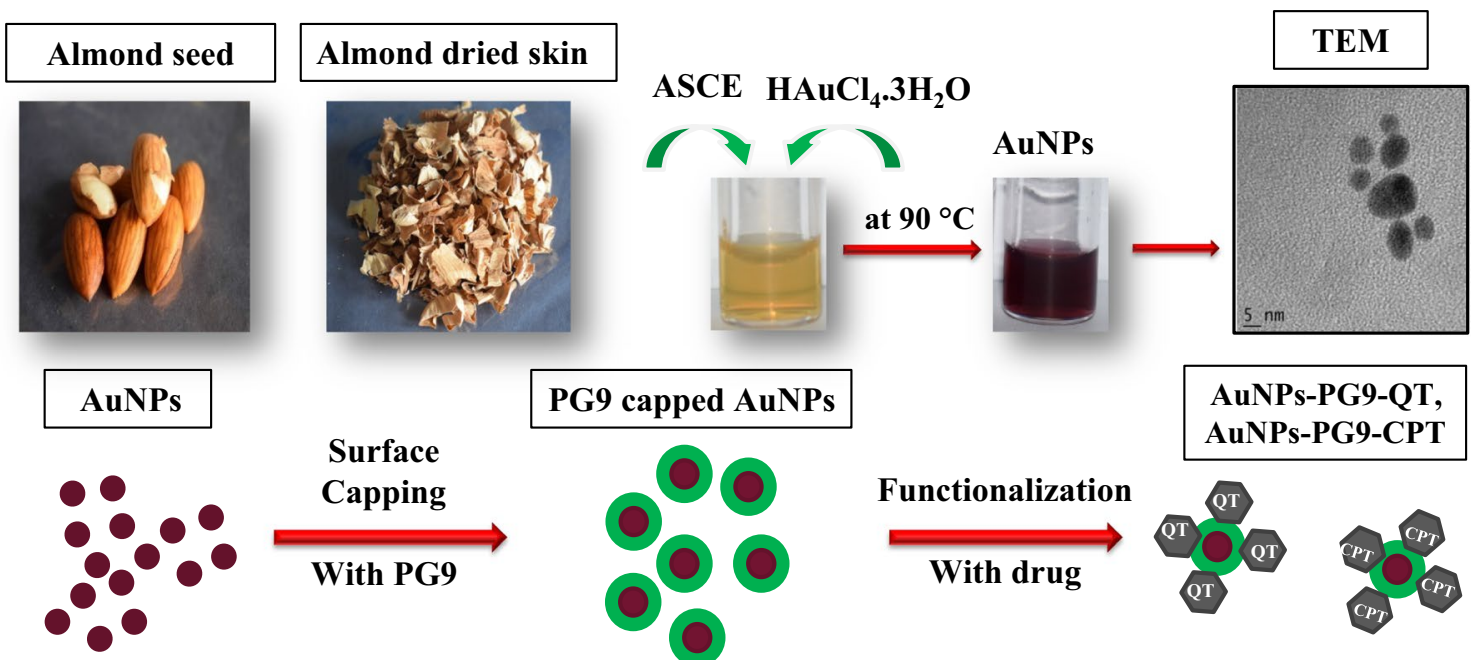

\section{Capping}

With PG9

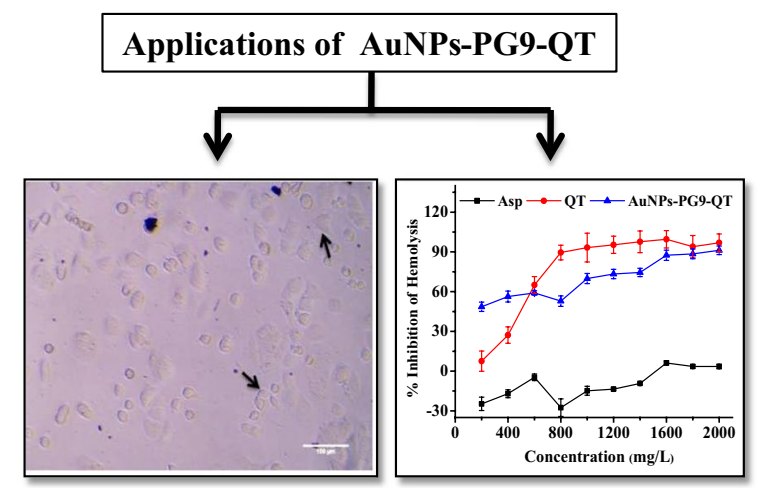

Anti-cancer activity Anti-inflammatory activity

biomedical application.
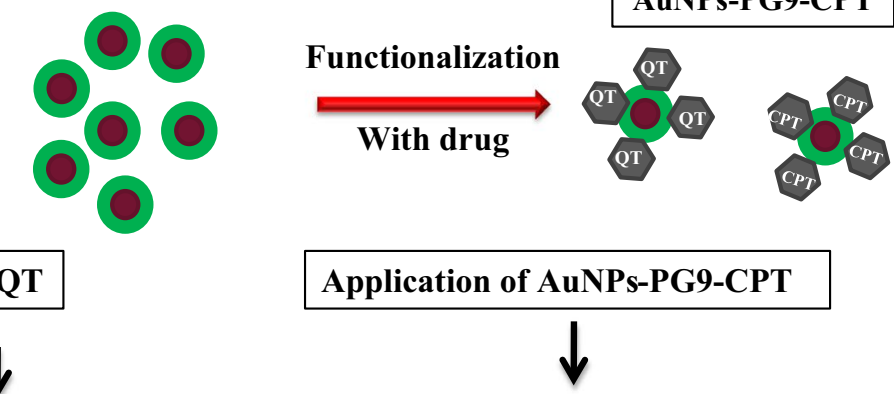

Keywords: Gold nanoparticles, Functionalization of capped Gold nanoparticles, Anti-inflammatory activity, Cytotoxicity, Anti-angiogenesis

\section{Background}

Every year, tens of millions of people around the world are diagnosed with cancer, and it is estimated that more than half of them do not survive [1]. As estimated by GLOBOCAN (2018), the number of new cancer cases will reach 18.1 million, and cancer-related deaths will be close to 9.6 million [2]. As population aging continues in many countries, cancer will remain a major health problem around the globe [1]. Mainly, breast cancer is the second leading cause of death in women [3]. Most of the available cancer therapies respond with undesirable side effects on healthy cells [4]. Therefore, the prominent challenge in the cancer treatment is to develop a targeted drug delivery system using carrier tools from natural sources [5]. The size and shape of the drug vectors are of particular importance for the successful development of the drug delivery system
[6]. Nanotechnology provides remarkable solutions to develop such tunable and suitable carrier platforms [7]. The development of nano delivery systems is a rapidly emerging area of nanotechnology application where nanomaterials (NMs) are employed to deliver therapeutic agents to a specific site in a controlled manner. In the recent times, the synthesis and use of NMs, especially metallic NMs have fascinated the scientific community as they are an excellent carriers for drugs [8], and can be modified in various ways for the controlled release of therapeutic payload into the cells for targeted delivery $[9,10]$. It has been proven that conjugates of nano-size can penetrate deeper into tumors, are efficiently endocytosed, and work against primary and metastatic tumors [11]. Recently, gold nanoparticles (AuNPs) have received a great deal of attention as nano-carriers due to their unique anti-cancer [12] and 
anti-angiogenic [13] properties. Moreover, properties of AuNPs such as biocompatibility and ease of surface functionalization make them suitable for biomedical imaging [14] and as carrier in targeted drug delivery systems [15]. Also, the variety of organic ligands can be functionalized on the surface of AuNPs to enhance their properties [13].

For the development of nano-size drug delivery system, NMs synthesized by various methods such as chemical, physical and biological methods can be explored. In comparison, biological methods are preferred as they depend on minimum use of harmful chemical and generate the minimum toxic by-products [16]. In addition, biogenic synthesis methods offer control over the size and shape of NMs, facilitate mass production and reproducibility [17]. Nanomaterials such as AuNPs biosynthesized using plant [18], bacteria [19], fungi [12], yeast [20], algae [21] etc. are generally safer for use in biomedical applications, especially in cancer treatment, since they come from natural materials themselves. Among these, the plant-based methods provide new avenues for cost-effective, simple and rapid synthesis of metal NPs (MNPs) due to readily availability of plant materials. In addition, plant based active principles such as curcumin has also been employed for the synthesis of AuNPs [22, 23]. Since plant metabolites are economical, non-toxic [24] and accelerate the reduction of metal ions to MNPs, they show good potential to fulfill the high demand for MNPs for use in the biomedical applications [25]. Also they are suitable for surface functionalization due to their small size and stability [26].

The present study demonstrates plant mediated, optimized biosynthesis of AuNPs for functionalization with anti-cancer drugs namely quercetin (QT) and camptothecin (CPT). To accomplish this, almond seed coat extract (ASCE) was employed to optimize the biogenic synthesis. The optimization was done by studying the effects of variations in different synthesis parameters such as incubation time, temperature, $\mathrm{pH}$, ratio of extract:precursor and precursor concentration. Next, the optimally synthesized AuNPs were characterized using various spectroscopic and imaging techniques, surface capped with polyethylene glycol 9000 (PG9) and then functionalized with QT and CPT. Further, anti-cancer and anti-inflammatory activities of QT functionalized AuNPs were evaluated against Human Breast Cancer Cell line MCF-7 (MCF7) using 3-(4,5-dimethylthiahiazol-2-yl)-2,5-diphenyl tetrazolium (MTT) assay and by inhibition of hemolysis of red blood cell (RBC) membrane, respectively. In addition, chorioallantoic membrane (CAM) based assay for the assessment of in vivo anti-angiogenic activity was employed to evaluate the potential of CPT functionalized
AuNPs to inhibit the formation of blood vessel in chick embryos.

\section{Results and discussion Optimization of biogenic synthesis of AuNPs}

Biogenic synthesis of MNPs is gaining importance for being simple, eco-friendly, lower toxicity and good biocompatibility of biogenic MNPs [27]. Since plants are easily available, safe to handle and consist of broad range of metabolites, they are preferred as sources of capping and reducing agents for biogenic synthesis [28]. In the present study, the initial reaction between ASCE and $\mathrm{HAuCl}_{4}$ precursor solution at $90^{\circ} \mathrm{C}$ for 10 min turned the reaction color from pale yellow to light purple and then dark ruby red indicating the synthesis of AuNPs due to reduction of $\mathrm{Au}^{+3}$ to $\mathrm{Au}^{\circ}$ (Fig. 1a). The ASCE mediated AuNPs exhibited a strong SPR peak at $530 \mathrm{~nm}$ which is in agreement with earlier few studies that reported phytomediated synthesis of AuNPs from Mangifera indica leaf [29], Magnolia kobus leaf extracts [30] and Solanum torvum [31]. The literature survey indicates that the optimization of different reaction parameters is needed to control the size and shape of the biogenic NMs [32-34]. Therefore, we next optimized the ASCE mediated AuNPs synthesis by studying the effects of variation in different reaction parameters on synthesis.

\section{Effect of incubation time on AuNPs synthesis}

Figure $1 \mathrm{~b}$ shows the effect of incubation time on ASCE mediated AuNPs synthesis. At 0 min, the reaction was pale yellow with a broad, hyperbolic SPR peak that indicated the slow interaction of biomolecules with precursor solution. As the reaction proceeded from 0 to $1 \mathrm{~min}$., color turned light purple due to ASCE mediated initiation of bio-reduction of $\mathrm{Au}^{+3}$ to AuNPs. Further continuation of reaction from 1 to 4 min gradually turned the reaction color from light purple to dark purple with broad SPR peak at $530 \mathrm{~nm}$ demonstrating AuNPs synthesis. Thereafter, the intensity of SPR peak at $530 \mathrm{~nm}$ and ruby red color gradually increased when incubation was extended from 5 to $8 \mathrm{~min}$ indicating the complete reduction (Fig. 1b). When incubation time was further increased to 9 and $10 \mathrm{~min}$, SPR peaks at $530 \mathrm{~nm}$ were found shifted to 541 and $548 \mathrm{~nm}$, respectively. Thus, the variation in reaction time clearly demonstrated that ASCE requires $1 \mathrm{~min}$ to initiate the reaction and completes in $8 \mathrm{~min}$. Therefore, the ASCE mediated AuNPs synthesis demonstrated in this study was rapid when compared with earlier studies $[27,35]$.

\section{Effect of temperature on AuNPs synthesis}

In addition to reaction time, the reaction temperature has also been proven as one of the important factors that 

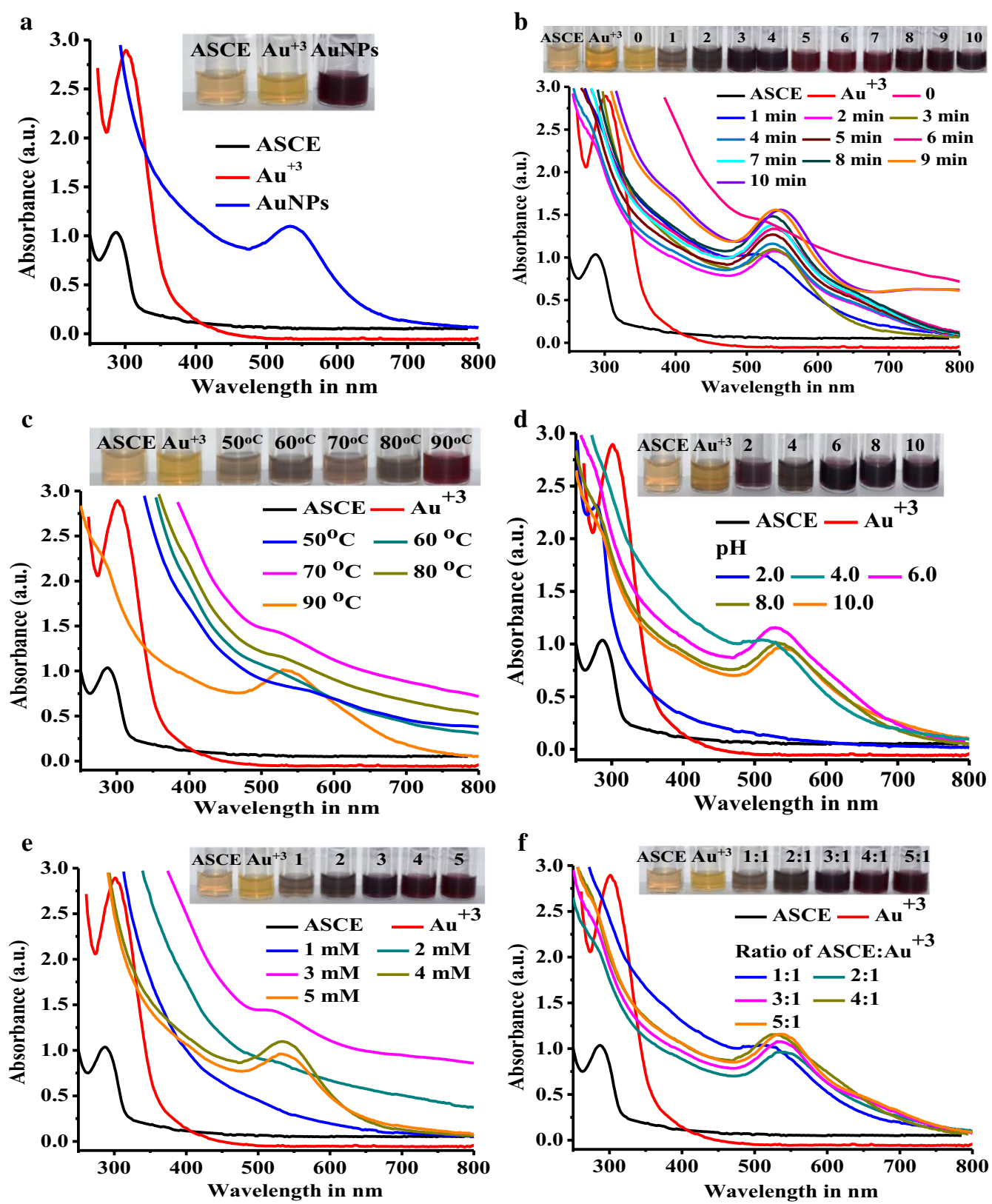

Fig. 1 Optimization of almond seed coat extract mediated biogenic synthesis of AuNPs. a Color change of initial biogenic synthesis reaction, and UV-Vis absorption spectra showing the effects of $\mathbf{b}$ incubation time, $\mathbf{c}$ incubation temperature, $\mathbf{d} \mathrm{pH}$, e concentration of $\mathrm{HAuCl}_{4}$ and $\mathbf{f}$ ratio of extract:precursor on biogenic synthesis of AuNPs

influence the rate of nucleation and reaction, growth, yield, and other properties of AuNPs [33]. As the temperature increases, the rate of reaction accelerates due to increase in kinetic energy and nucleation resulting in the formation of small nuclei [30] whereas, low temperature requires more time for AuNPs synthesis [34]. In the view of this effect of reaction temperature, we varied the reaction temperature in the range of $50-90{ }^{\circ} \mathrm{C}$ for optimum AuNPs synthesis (Fig. 1c). On incubating the reactions in the range of $50-80^{\circ} \mathrm{C}$, no dark ruby red color with sharp SPR peaks characteristic of AuNPs were observed (Fig. 1c). This suggested that at $50-80^{\circ} \mathrm{C}$, there was incomplete or slow reduction of $\mathrm{Au}^{+3}$ ions to $\mathrm{Au}^{\circ}$. Noteworthy, when the reaction was incubated at $90^{\circ} \mathrm{C}$, it turned dark ruby red and showed typical SPR at $530 \mathrm{~nm}$ indicating the optimum reduction of $\mathrm{Au}^{+3}$ to smaller 
sized AuNPs (Fig. 1c). Our observation coincides with previous few studies that also reported the optimum biogenic syntheses of AuNPs at higher temperatures using extracts of various plants such as Tiliacorat riandra [36], Magnolia kobus [30] and Chenopodium album [37].

\section{Effect of $\mathrm{pH}$ on AuNPs synthesis}

It is known that $\mathrm{pH}$ of the synthesis reaction also plays a substantial role in reduction, synthesis and stabilization of NPs. It can be explained by the fact that increase in the reaction $\mathrm{pH}$ promotes the capping of NP's surface more efficiently and supports the formation of smaller NPs [38]. In the present study, when precursor solution was added to ASCE and $\mathrm{pH}$ of the reaction mixture was measured before embarking into reaction, it was found to be 6 . Therefore, for the study of influence of $\mathrm{pH}$, we studied the effect of variation of $\mathrm{pH} 6 \pm 4$ i.e. in the range of 2-10 (Fig. 1d). At high acidic $\mathrm{pH} 2$, synthesis was not observed whereas, at $\mathrm{pH} 4$, reaction turned slightly purple but no characteristic SPR peak of AuNPs was observed. This indicated that high acidic condition did not favor the synthesis of AuNPs as it could have inactivated the biomolecules of ASCE functioning as reducing and capping agents. This was quite in congruence with the observation made by Pimprikar and co-workers (2009). These authors demonstrated that the extreme acidic conditions $(\mathrm{pH} 2)$ of reaction inactivate the metabolites of extract and are likely to reduce the synthesis [20]. In our case, when the $\mathrm{pH}$ was increased to slight acidic condition of 6 , the reaction changed from light yellow to dark ruby red with intense and narrow SPR peak at $529 \mathrm{~nm}$ which clearly demonstrated AuNPs synthesis (Fig. 1d). Though further increase in $\mathrm{pH}$ to 8 and 10 turned reactions dark ruby red, the SPR peaks were broadened and accompanied with red shifts at 540 and $545 \mathrm{~nm}$ respectively. Moreover, intensities of the SPR peaks were reduced demonstrating the synthesis of large sized AuNPs. The observed red shifts in reactions at alkaline $\mathrm{pH}$ could have occurred due to plasmon coupling between aggregated NPs [39]. Thus, we found that $\mathrm{pH}$ 6 was optimum for ASCE mediated synthesis of AuNPs.

\section{Effect of precursor concentration on AuNPs synthesis}

In order to study how the variation in the precursor $\mathrm{HAuCl}_{4}$ concentration influences the AuNPs synthesis, it was varied in the range of 1-5 mM and analyzed (Fig. 1e). Precursor in the range of 1-3 mM turned reactions light yellow but AuNPs were not synthesized as no sharp and intense characteristic SPR peaks were observed. However, when reaction contained $4 \mathrm{mM} \mathrm{HAuCl}_{4}$, dark ruby red color was observed with sharp and intense SPR peak at $530 \mathrm{~nm}$ indicating complete reduction of $\mathrm{Au}^{+3}$ ions to AuNPs (Fig. 1e). In case of use of $5 \mathrm{mM}$ of $\mathrm{HAuCl}_{4}$, the SPR peak was broadened and slightly red shifted to $536 \mathrm{~nm}$ with decreased intensity. These results are in agreement with the previous report that states that the concentration of biomolecules of plant extract may not be sufficient to reduce high concentration of $\mathrm{HAuCl}_{4}$ [20].

\section{Effect of extract to precursor ratio on AuNPs synthesis}

In addition to incubation time, temperature, $\mathrm{pH}$ and concentration of precursor, the ratio of extract to precursor is also known to influence the synthesis of MNPs such as AuNPs [20]. In the light of this information, we varied the ASCE:precursor ratio in the range of 1:1-5:1 and analyzed its effect of AuNPs synthesis (Fig. 1f). At 1:1 ratio, the reaction turned light purple without distinct SPR peak at $530 \mathrm{~nm}$. Later, when ratio was increased to $2: 1$ and 3:1, it developed a light and dark purple color with broad SPR peaks at 540 and $537 \mathrm{~nm}$, respectively whereas, the SPR peak of reaction with 4:1 ratio was found proportionally more intense at $530 \mathrm{~nm}$ indicating the optimum synthesis (Fig. 1f). In case of 5:1 ratio, the intense SPR peak was shifted to $537 \mathrm{~nm}$ demonstrating the synthesis of large sized AuNPs. Our observation of increased NPs size due to increased concentration of extract is in agreement and goes well with earlier report. Khalil and colleagues found that the reaction with higher volumes of the extract shows slightly decreased intensity and red shift of SPR indicating the saturation in the reduction of $\mathrm{Au}^{+3}$ [38].

Thus, based on these studies of effects of variation in different synthesis parameters, it was found that AuNPs were optimally synthesized at $8 \mathrm{~min}$ of reaction, $90{ }^{\circ} \mathrm{C}$, $\mathrm{pH}$ 6, $4 \mathrm{mM}$ of $\mathrm{HAuCl}_{4}$ and 4:1 ratio of ASCE: $\mathrm{HAuCl}_{4}$.

\section{Characterization of AuNPs}

For the characterization of AuNPs using elemental analysis, the recording of EDS spectra illustrated the presence of elemental gold ( $\mathrm{Au}$ ) (Fig. 2a). The EDS profile showed a strong signal of $\mathrm{Au}$ followed by carbon $(\mathrm{C})$, oxygen (O) and minor peak of polonium due to AuNPs surface bound organic biomolecules from ASCE [40]. Gold peak was observed approximately at $2.2 \mathrm{keV}$ which is typical for the absorption of metallic Au nano-crystallites due to SPR. The EDS profile demonstrated that Au contributed by 79.52 weight \% to AuNPs whereas other detected elements namely $\mathrm{C}$ and $\mathrm{O}$ were found to contribute by 14.75 and 6.47 weight $\%$, respectively. The higher elemental contribution by Au clearly demonstrated that synthesized NPs were indeed AuNPs whereas, the presence of $C$ and O related signal confirmed the biogenic nature of AuNPs.

The FTIR spectra of ASCE (Fig. 2b) showed strong band of IR stretchings at 3219.01 and $2091.00 \mathrm{~cm}^{-1}$ corresponding to $\mathrm{OH}$ groups bonded to the aromatic 

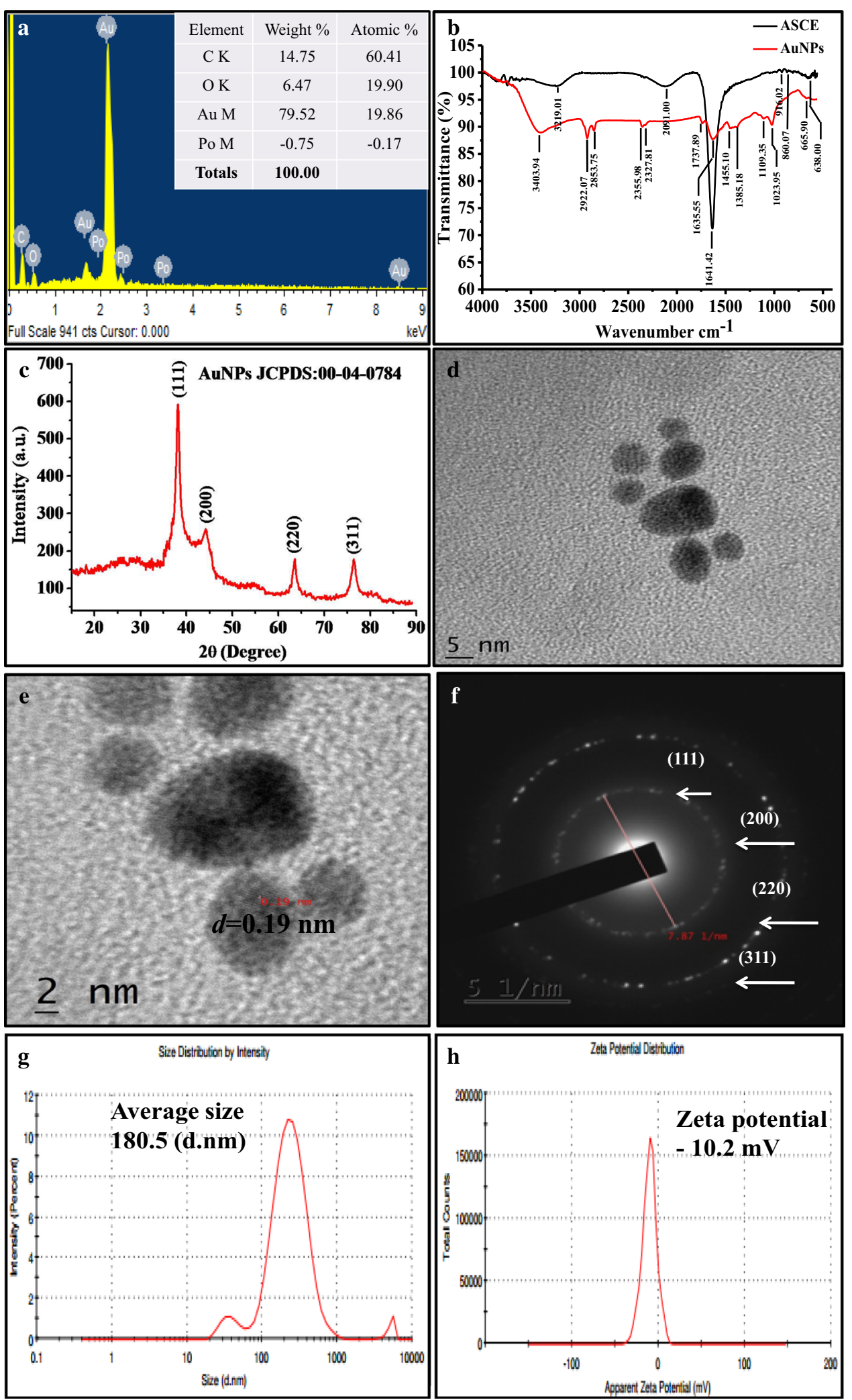

Fig. 2 Characterization of biogenic AuNPs by a EDS, b FTIR, c XRD, d TEM, e HR-TEM, f SAED, $\mathbf{g}$ DLS and $\mathbf{h}$ Zeta potential pattern analyses 
ring and $\mathrm{C}-\mathrm{C}$ stretching, respectively (Additional file 1 : Table S1) whereas, IR band at $1641.42 \mathrm{~cm}^{-1}$ in the spectrum of ASCE and at $1635.55 \mathrm{~cm}^{-1}$ in IR spectrum of AuNPs were assigned to $\mathrm{N}-\mathrm{H}$ bending vibration of primary amines. The capping of ASCE biomolecules onto the AuNPs surface could have broken most of the $\mathrm{H}$ bonds between the $\mathrm{N}-\mathrm{H}$ groups that could have led to observed marginal shift [27] and decrease in intensity of IR band [41]. The FTIR spectra of AuNPs showed the major characteristic peak at $3403.94 \mathrm{~cm}^{-1}$ related to $\mathrm{O}-\mathrm{H}$ and $\mathrm{N}-\mathrm{H}$ stretching in amine group while other peaks at 2922.07 and $2853.75 \mathrm{~cm}^{-1}$ were attributed to $\mathrm{C}-\mathrm{H}$ stretching vibrations. The intense broad absorbance at 2355.98 and $1023.95 \mathrm{~cm}^{-1}$ corresponded to $\mathrm{C}=\mathrm{C}$ and $\mathrm{C}-\mathrm{N}$ stretching vibration of aliphatic amines or to alcohols/phenols, respectively. The emergence of new peak at $1737.89 \mathrm{~cm}^{-1}$ corresponding to stretching vibrations of carbonyl $(\mathrm{C}=\mathrm{O})$ indicated the conversion of alcoholic group into aldehyde to reduce $\mathrm{Au}^{+3}$ to $\mathrm{Au}^{\circ}$ [42]. The additional minor peaks of AuNPs corresponded to different bending and stretching vibrations of the bioactive molecules as shown in Additional file 1: Table S1. Thus, our FTIR based investigation indicated the involvement of amines, alcohols, phenols, and aromatic groups of ASCE as reducing and capping agents in synthesis of AuNPs.

The recording and analysis of XRD pattern (Fig. 2c) of AuNPs for the study of crystalline nature showed four intense peaks of $2 \theta$ values in the range from 10-90. These four distinct diffraction peaks at $36.58^{\circ}, 42.71^{\circ}, 61.90^{\circ}$ and $74.78^{\circ}$ corresponded to (111), (200), (220) and (311) crystallographic planes. The Bragg reflections closely matched to the reported reference values of Joint Committee on Power Diffraction Standards (JCPDS) card No. 04-0784 which demonstrated that AuNPs resembled the face-centered cubic (FCC) structure of metallic $\mathrm{Au}$ and composed of pure crystalline Au. The observed XRD pattern coincided with that previously reported by Rajakumar et al., (2016) who stated that AuNPs with FCC structure were oriented along the (111) plane[18].

Further, imaging the morphologies and size by TEM indicated the spherical and oval shape of AuNPs with an average size in the range of 5-15 $\mathrm{nm}$ (Fig. 2d). The HR-TEM analysis also demonstrated and confirmed the spherical morphology and internal polycrystalline nature of AuNPs with $0.19 \mathrm{~nm}$ of $\mathrm{d}$-spacing in the lattice fringes (Fig. 2e). The TEM based SAED pattern (Fig. 2f) showed diffraction rings associated with the (111), (200), (220) and (311) atomic planes. Spots corresponding to various orientations of the nanocrystals which appeared inside the concentric rings clearly showed crystalline nature of AuNPs that matched with XRD pattern as shown in Fig. 2c. Thus, the HR-TEM and SAED analyses clearly confirmed the single crystal structure of AuNPs with FCC phase. The size of AuNPs and their distribution in solution analyzed by DLS (Fig. $2 \mathrm{~g}$ ) indicated the polydispersed nature of AuNPs with average hydrodynamic diameter of $180.5 \mathrm{~nm}$ (d.nm). The average zeta potential of -10.2 (Fig. $2 \mathrm{~h}$ ) $\mathrm{mV}$ showed good stability and negative charge on AuNPs surface due to capping by biomolecules of ASCE.

\section{Surface capping of AuNPs}

In the present study, our initial attempts to functionalize neat AuNPs with QT and CPT failed as they were not efficiently functionalized. This could have occurred due to the lack of suitable surface groups or ligands for exchange with these drugs. Therefore, in order to facilitate efficient functionalization, we first surface capped AuNPs with polymers such as PG6, PG9 and PVP K25. Additional file 1: Figure S1 indicate the UV-Vis absorption spectra of surface capping reactions of AuNPs with PG6, PG9 and PVP K25. The reactions of AuNPs with tested capping agents resulted in the red shift of absorption bands in the range of 535-538 $\mathrm{nm}$. The resultant slight decrease in color intensities indicated the modifications in the AuNPs surface due to surface capping (Additional file 1: Fig. S1). It was observed that AuNPs surfaced capped with PG9 displayed a characteristic peak at $535 \mathrm{~nm}$ with high absorbance as compared to AuNPs surfaced capped with PG6 and PVP K25 (Additional file 1: Fig. S1). These peaks related to the surface plasmon and increase in intensity was observed due to increased density of the reaction mixture by capping with polymers (Additional file 1: Fig. S1). Similar effect of capping agents has been reported by $\mathrm{Oh}$ and coworkers [43]. It was reported that red shift of SPR band in capping reaction is a consequence of increased refractive index surrounding the AuNPs due to the modification of PEG molecular chain length [44]. Moreover, the slight variation in absorption values of AuNPs capped with PG6 (Additional file 1: Fig. S1) and PG9 (Additional file 1: Fig. S1) indicated that AuNPs were coated with PEG of different molecular weight [45]. Considering these, it was concluded that PG9 was comparatively more suitable for surface capping of AuNPs and, therefore was selected for further optimization of functionalization with anti-cancer drugs.

\section{Optimization of functionalization of AuNPs-PG9 with QT and CPT}

Though the initial reaction between PG9 capped AuNPs (AuNPs-PG9) and QT solution at $50{ }^{\circ} \mathrm{C}$ for $2 \mathrm{~h}$ changed the reaction from dark ruby red to light ruby red with strong SPR peak at $536 \mathrm{~nm}$ indicating functionalization (Additional file 1: Fig. S2a), it was worth investigating 
how the variation in different reaction conditions influences the functionalization. Additional file 1: Figure S2 represents the effect of incubation time on functionalization of QT with AuNPs-PG9. It was found that increase in the reaction time was accompanied by increase in SPR peak intensity. The SPR peak intensity of functionalization reaction was gradually increased with increased reaction time from 0 to $30 \mathrm{~min}$ (Additional file 1: Fig. S2b). Further increase from 45 to $60 \mathrm{~min}$ also resulted in increased absorbance with sharp, narrow SPR peak at 535 and $533 \mathrm{~nm}$, respectively. After $60 \mathrm{~min}$ of reaction (75-120 min), the narrow SPR peak started broadening with reduced SPR peak intensities. Previously, it has been reported that inter-particle spacing decreases with increased reaction time that subsequently leads to slight red shift indicating surface functionalization [46]. Therefore, in our case, $60 \mathrm{~min}$ was found optimum reaction time for functionalization of QT with AuNPs-PG9.

The variation in reaction $\mathrm{pH}$ in range of 4-7 demonstrated that $\mathrm{pH} 4$ and 5 result in red shift and broad SPR peak $(539 \mathrm{~nm})$ whereas; $\mathrm{pH} 6$ and 7 result in gradual increase in SPR intensity with blue shift (Additional file 1: Fig. S2c). It has been stated that QT functionalized with NPs exhibit maximum stability at pH between 6 and 7 . Within this $\mathrm{pH}$ range, the interaction between AuNPsPG9 and QT occurs through hydrogen bond formation since both maybe deprotonated and protonated in alkaline and acidic condition, respectively; that may influence the H-bonding [47].

In addition to variation in incubation time and $\mathrm{pH}$ of the reaction, the effect of variation in QT concentration on functionalization was also studied by varying the AuNPs-PG9:QT ratio in the range of 1:1-1:4 and monitored by UV-Vis. spectroscopy (Additional file 1: Fig. S2d). When ratio was increased from 1:1 to 1:4, the SPR peak intensities linearly increased with slight red shifts from 530 to $535 \mathrm{~nm}$. It is stated that the lower concentration of molecules to be functionalized does not effectively built-up around the NPs whereas, high enough concentration favors the functionalization of surface capped NPs [48]. Since highest SPR peak intensity with red shift was noted for reaction with ratio 1:4, it was consider optimum for preparation of AuNPs-PG9 functionalized with QT (AuNPs-PG9-QT).

The effect of variation of reaction temperature in range of $30-70{ }^{\circ} \mathrm{C}$ on AuNPs-PG9-QT synthesis resulted in comparatively less intense and broad SPR peaks at 30 and $50{ }^{\circ} \mathrm{C}$ (Additional file 1: Fig. S2e). This may be due to the increased size and hydrogen bond formation between AuNPs-PG9 and QT [49]. On the contrary, more intense and sharp SPR band was observed at $533 \mathrm{~nm}$ when reaction was incubated at $70{ }^{\circ} \mathrm{C}$ indicating it as the optimum temperature for AuNPs-PG9-QT synthesis.
Besides optimizing the AuNPs-PG9:QT ratio, the effect of variation in the concentration of QT in the range of 400-1600 ppm was also investigated in the present study (Additional file 1: Fig. S2f). We observed that SPR intensity was gradually increased when QT concentration was increased from 400 to $1200 \mathrm{ppm}$. At QT $>1200 \mathrm{ppm}$ i.e. in the range of $1400-1600 \mathrm{ppm}$, the SPR bands turned broad with slight red shifts. The broadening and shifting of absorption band could have occurred due to the formation hydrogen bonds in AuNPs clusters [50]. The highly intense and sharp SPR band was recorded when reactions contained $1200 \mathrm{ppm}$ of QT; therefore, we considered it as the best concentration for AuNPs-PG9-QT synthesis. Thus, based on variations in the different conditions of functionalization reaction, we found that incubation time of $60 \mathrm{~min}, \mathrm{pH}$ 7, AuNPs-PG9:QT ratio of 1:4, temperature of $70{ }^{\circ} \mathrm{C}$ and $1200 \mathrm{ppm}$ of QT were the most optimum conditions for functionalization of AuNPs-PG9 with QT.

In addition to functionalization with QT, AuNPs-PG9 were also tested for their potential to get functionalized with CPT. When the effect of concentration of CPT in the range of $0.1-0.5 \mathrm{mM}$ was tested, $0.5 \mathrm{mM}$ CPT resulted in higher SPR intensity and shift to $535 \mathrm{~nm}$ indicating the optimum functionalization (Additional file 1: Fig. S3a). Further, optimization of reaction time in the range of $1-6 \mathrm{~h}$ indicated $1 \mathrm{~h}$ as optimum time as SPR peak was found shifted to $536 \mathrm{~nm}$. This SPR shift clearly demonstrated that $1 \mathrm{~h}$ reaction time was optimum for functionalization of CPT with AuNPs-PG9 (Additional file 1: Fig. S3b). Likewise, variation of $\mathrm{pH}$ of the reaction in the range of 2-12 showed that the optimum functionalization of CPT takes place in basic condition ( $\mathrm{pH} 12)$, since the reaction showed the shift of SPR to $539 \mathrm{~nm}$ (Additional file 1: Fig. S3c).

Thus, based on variations in the different conditions, we found that incubation time of $1 \mathrm{~h}, \mathrm{pH} 12$, AuNPsPG9:CPT ratio of $1: 1, \mathrm{RT}$ and $0.5 \mathrm{mM}$ of CPT were the most optimum conditions for functionalization of AuNPs-PG9 with CPT.

\section{Characterization of AuNPs-PG9-QT and AuNPs-PG9-CPT}

The large scale synthesis of AuNPs-PG9-QT and its comparison with neat AuNPs and AuNPs-PG9 by UVVis spectroscopy showed typical SPR peaks at 535,530 and $531 \mathrm{~nm}$, respectively (Fig. 3a). The elemental analysis of AuNPs-PG9-QT by EDS (Fig. 3b) exhibited the typical signal of metallic Au nano-crystallites at $2.2 \mathrm{keV}$. When compared with EDS of neat AuNPs, the EDS spectrum of AuNPs-PG9-QT showed that elemental $\mathrm{Au}$ accounted for 22.79 weight \%. Noteworthy, $\mathrm{C}$ and $\mathrm{O}$ contents were found increased from 14.75 and $6.47 \%$ in AuNPs (Fig. 2a) to 43.73 and $33.48 \%$ in AuNPs-PG9-QT 


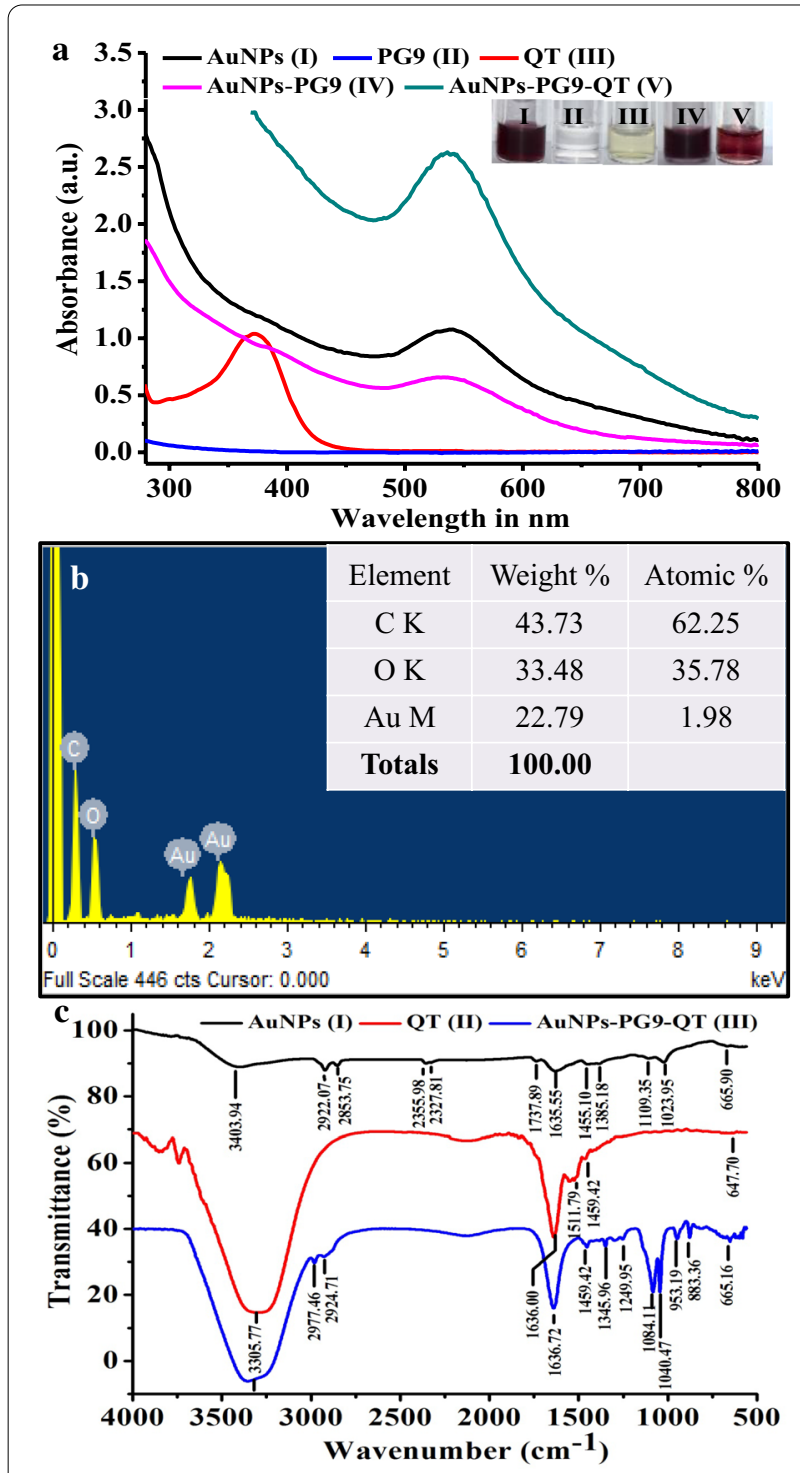

Fig. 3 Characterization of polyethylene glycol 9000 capped and quercetin functionalized AuNPs by $\mathbf{a} U V-V i s, \mathbf{b}$ EDS and $\mathbf{c}$ FTIR spectroscopy

(Fig. 3b), respectively. The increased contents of $\mathrm{C}$ and $\mathrm{O}$ complex suggested that considerable amount of $\mathrm{Au}$ atoms were capped, covered with large number of QT molecules demonstrating successful functionalization. The similar changes in EDS spectrum of functionalized NPs has been reported previously [47]. Further analysis of AuNPs-PG9-QT by FTIR confirmed the functionalization as several bands present in the IR spectra of AuNPs and QT (Fig. 3c) were shifted, however their characteristic signatures were maintained. The FTIR spectrum of AuNPs-PG9-QT (Fig. 3c) showed characteristic peaks at $3305.77 \mathrm{~cm}^{-1}$ corresponding to free $\mathrm{OH}$ bond vibration whereas, peaks at 2977.46 and $2924.71 \mathrm{~cm}^{-1}$ were assigned to the stretching vibration of $\mathrm{C}-\mathrm{H}$ suggesting the inter-molecular hydrogen bonding between QT and PG9 capped AuNPs. In addition, other major and minor characteristic peaks were also detected in AuNPs-PG9-QT in the range of $1600-650 \mathrm{~cm}^{-1}$. The stretching vibrations of these peaks and their functional groups are presented in Additional file 1: Table S2. The absorption band representing aromatic group of QT (at $1511.79 \mathrm{~cm}^{-1}$ ) was completely disappeared in spectrum of AuNPs-PG9QT. The IR band at $1249.95 \mathrm{~cm}^{-1}$ in FTIR spectrum of AuNPs-PG9-QT (Fig. 3c) could be attributed to $\mathrm{C}-\mathrm{O}$ stretching vibrations suggesting the formation of ester bond for binding of carboxyl end of PG9 with the catechol group of QT [51]. Moreover, presence of C-O, $\mathrm{C}=\mathrm{O}, \mathrm{C}-\mathrm{H}$ and $\mathrm{N}-\mathrm{H}$ related stretching vibration confirmed the presence of bound PG9. The prominent IR peaks related to phenol and aromatic group represented the effective binding of QT to form AuNPs-PG9-QT conjugate (Fig. 3c and Additional file 1: Table S2) which is well in agreement with the existing data in the literature [52].

In case of characterization of AuNPs-PG9-CPT by EDS, highest peak corresponding to AuNPs was recorded at $2.2 \mathrm{keV}$ while other peaks at $0.2,0.4$ and $1 \mathrm{KeV}$ corresponding to $\mathrm{C}, \mathrm{O}$ and $\mathrm{Na}$ respectively were also recorded. Based on EDS analysis, Au was found to contribute by $60.49 \%$ to the weight of AuNPs-PG9-CPT (Fig. 4a). The FTIR spectrum of AuNPs-PG9-CPT showed different IR peaks at different positions for various functional groups. The peak at $1735 \mathrm{~cm}^{-1}$ corresponded to carbonyl stretching for cyclic lactone while the band at $1143 \mathrm{~cm}^{-1}$ corresponded to $\mathrm{C}-\mathrm{C}(=\mathrm{O})-\mathrm{O}$ stretching vibration. In addition, the IR band at $1618 \mathrm{~cm}^{-1}$ was ascribed to $\mathrm{C}=\mathrm{O}$ stretching vibration whereas the peaks at 2852, 2361 and $1095 \mathrm{~cm}^{-1}$ were assigned to $\mathrm{C}=\mathrm{H}$ stretching, $\mathrm{N}=\mathrm{C}=\mathrm{O}$ stretching and $\mathrm{C}=\mathrm{O}$ stretching, respectively. Also, the band at $2916 \mathrm{~cm}^{-1}$ was found to correspond to $\mathrm{C}-\mathrm{H}$ vibrational stretching of the methylene groups of the protein (Fig. 4b).

\section{Anti-cancer activity of AuNPs-PG9-QT}

In order to assess how the functionalization of QT with AuNPs-PG9 influences the anti-cancer potential, the anti-cancer activities of AuNPs, QT and AuNPs-PG9QT were studied by employing MTT assay on cancer cell line MCF-7 and compared. To this end, AuNPs-PG9QT in the range of $20-800 \mu \mathrm{g} / \mathrm{mL}$ were treated with MCF-7 cells and compared with untreated (control) cells (Fig. 5e). In comparison to AuNPs and QT which showed marginal activity, AuNPs-PG9-QT in the tested range significantly reduced the cell viability indicating good anti-cancer activity with $\mathrm{IC}_{50}$ value of $687.44 \mu \mathrm{g} /$ 

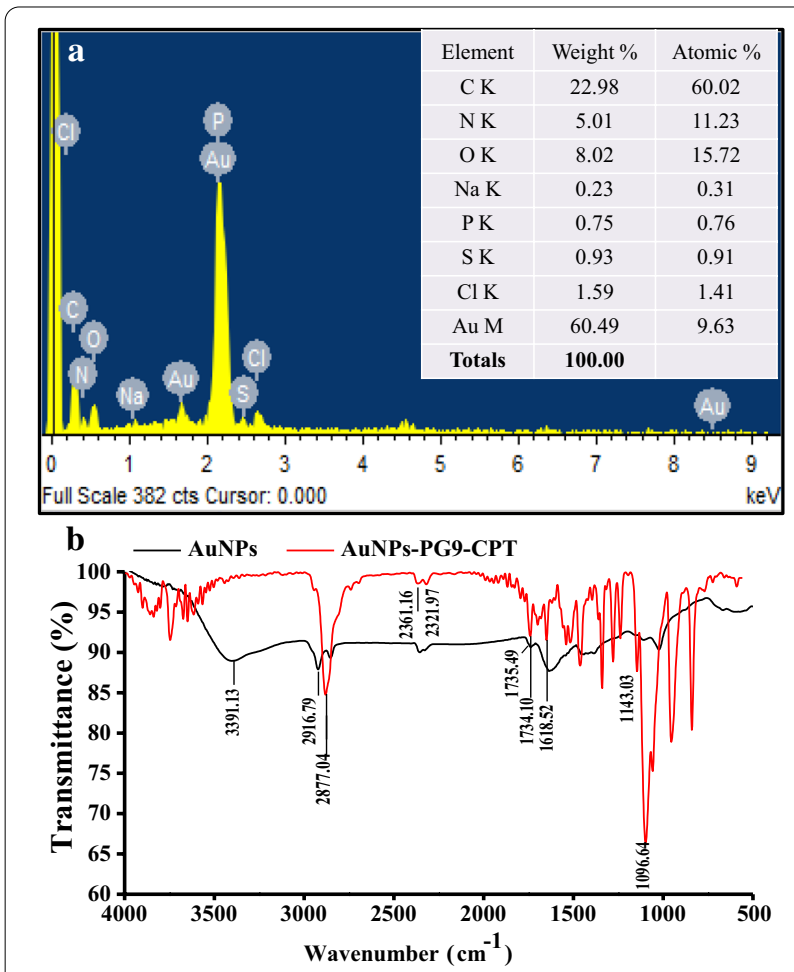

Fig. 4 Characterization of polyethylene glycol 9000 capped and camptothecin functionalized AuNPs by $\mathbf{a}$ EDS and $\mathbf{b}$ FTIR spectroscopy

$\mathrm{mL}$ (Table 1). This observation demonstrated that the functionalization of QT with AuNPs-PG9 does not reduce or loses the activity; rather the activity of functionalized preparation (AuNPs-PG9-QT) was enhanced significantly. In line with our observation, recently Rameshthangam and Chitra had also observed that the anti-cancer activity of PEG capped nickel NPs against MCF-7 cell line enhances when functionalized with QT [52]. Similar enhanced anti-cancer activity for arginine encapsulated PEG-coated magnetic has also been reported [53]. In the present study, the anti-cancer activity of AuNPs-PG9-QT was also evident from the change in morphologyof MCF-7 cells (Fig. 5a-d). As compared to untreated cells, AuNPs-PG9-QT treated cells were ruptured, few changed their shape from spindle to circular or irregular, and the number of adhered cells was also decreased (Fig. 5d). Our observation on changed cell morphology due to treatment of QT functionalized NPs is in agreement with that of Firoozeh et al. who studied the effect of QT encapsulated in solid lipid NPs [54].

\section{Anti-inflammatory activity ofAuNPs-PG9-QT}

Protein denaturation is well familiar cause for inflammation. Salicylic acids, flufenamic acid and other antiinflammatory drugs have shown dose dependent ability
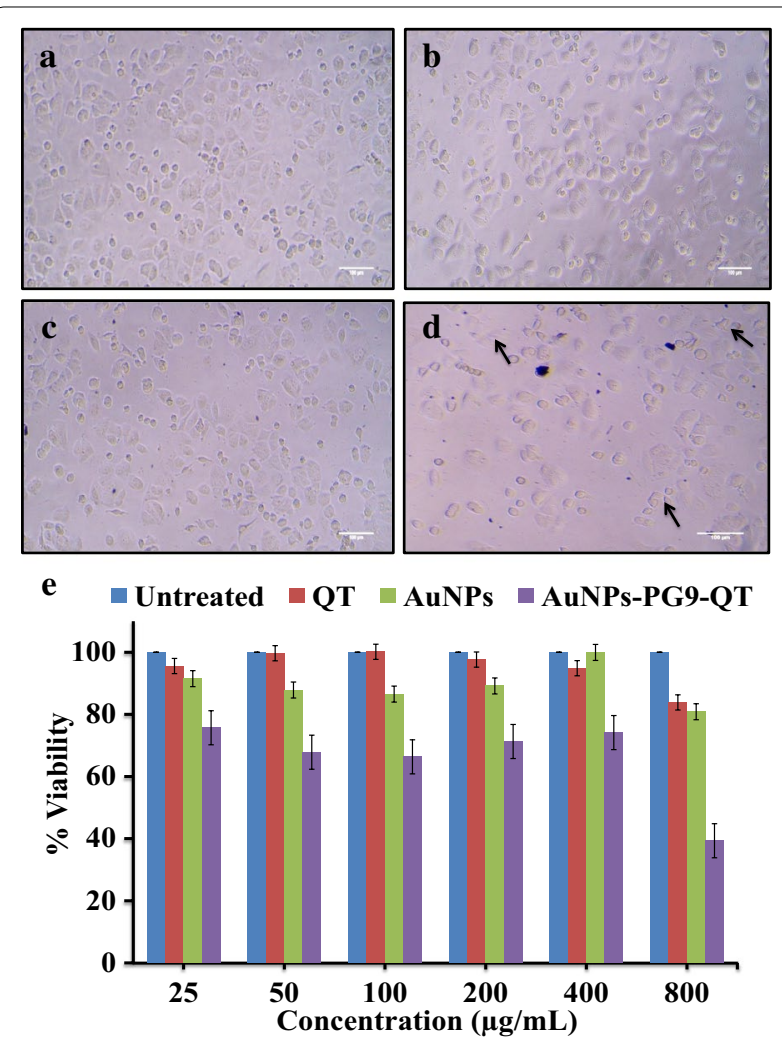

Fig. 5 Anticancer effect of polyethylene glycol 9000 capped and quercetin functionalized AuNPs. Morphological changes to MCF-7 cell line after treatment with $\mathbf{a}$ control, $\mathbf{b}$ only quercetin, $\mathbf{c}$ neat AuNPs, d polyethylene glycol 9000 capped Quercetin functionalized AuNPs and e effect of different concentration of Quercetin, AuNPs, and polyethylene glycol 9000 capped and Quercetin functionalized AuNPs on cell viability of MCF-7 cell line. (In $\mathbf{d}$ arrows indicate that AuNPs-PG9-QT treated cells were ruptured and observed for irregular shape)

Table 1 Effect of PG9 capped and quercetin functionalized AuNPs on toxicity to MCF-7 cell line

\begin{tabular}{ll}
\hline Sample & MCF-7 cell line $\mathrm{IC}_{50} 24 \mathrm{~h}$ \\
\hline Quercetin & NA (Low toxicity at highest concentration) \\
Biogenic AuNPs & NA (Low toxicity at highest concentration) \\
PG9 capped and Quercetin & $687.44 \mu \mathrm{g} / \mathrm{mL}$ \\
functionalized AuNPs & \\
\hline
\end{tabular}

to inhibit protein denaturation and RBCs membrane stabilization [55]. As a part of our efforts to investigate antiinflammatory potential, the activity of AuNPs-PG9-QT was studied to inhibit the heat induced hemolysis of RBCs and RBCs membrane stabilization. In this case, the standard drug Asp and control QT at $1600 \mathrm{mg} / \mathrm{L}$ showed a maximum of 6.18 and $99.51 \%$ inhibitions, respectively while AuNPs-PG9-QT in the range of 200-2000 mg/L 
Table 2 Effect of concentration of aspirin, quercetin and PG9 capped and quercetin functionalized AuNPs on the \% inhibition of hemolysis

\begin{tabular}{|c|c|c|c|}
\hline \multirow{2}{*}{$\begin{array}{l}\text { Concentration } \\
\text { (ppm) }\end{array}$} & \multicolumn{3}{|l|}{$\%$ Inhibition } \\
\hline & Aspirin & Quercetin & AuNPs-PG9-QT \\
\hline 200 & $-24.70 \pm 5.03$ & $7.50 \pm 7.64$ & $48.60 \pm 8.50$ \\
\hline 400 & $-17.02 \pm 2.98$ & $27.22 \pm 6.22$ & $56.33 \pm 15.09$ \\
\hline 600 & $-4.68 \pm 2.49$ & $65.05 \pm 6.36$ & $59.08 \pm 1.65$ \\
\hline 800 & $-27.60 \pm 6.66$ & $89.51 \pm 5.49$ & $52.86 \pm 3.97$ \\
\hline 1000 & $-14.81 \pm 3.4$ & $93.27 \pm 10.89$ & $69.90 \pm 3.77$ \\
\hline 1200 & $-13.58 \pm 0.91$ & $95.36 \pm 6.53$ & $73.35 \pm 8.51$ \\
\hline 1400 & $-9.26 \pm 0.74$ & $97.62 \pm 8.19$ & $74.51 \pm 21.16$ \\
\hline 1600 & $6.18 \pm 1.51$ & $99.51 \pm 6.55$ & $87.46 \pm 6.83$ \\
\hline 1800 & $3.51 \pm 1.3$ & $93.97 \pm 8.41$ & $88.52 \pm 8.67$ \\
\hline 2000 & $3.48 \pm 1.61$ & $96.85 \pm 6.67$ & $91.26 \pm 6.25$ \\
\hline IC50 & $-14.195 \pm 2.15$ & $94.315 \pm 8.71$ & $71.62 \pm 6.14$ \\
\hline
\end{tabular}

was found effective in inhibiting the heat induced hemolysis of RBS to variable extent (Table 2). With increase in the concentration of AuNPs-PG9-QT, the dose dependent inhibition of hemolysis was observed (Fig. 6a). Highest of $91.26 \%$ inhibition was achieved at $2,000 \mathrm{mg} / \mathrm{L}$ of AuNPs-PG9-QT with $\mathrm{IC}_{50}$ value of 287.177 (Fig. 6b). Although the precise mechanism of NPs mediated membrane stabilization is yet to be elucidated, it may be possible that AuNPs-PG9-QT produced this effect by changing the surface area/volume ratio of the cells. This could have caused an expansion of membrane or the shrinkage of the cells and an interaction with membrane proteins [56].

\section{Anti-angiogenic activity of AuNPs-PG9-CPT}

Angiogenesis is the process of formation of new blood vessels of tissue or organ and anti-angiogenic effects of test samples can be studied by assessing the test sample mediated prevention of growth of new blood vessels [57]. The chicken embryo is well-known animal model and the CAM based assay is widely used [58] as it effectively responds to pro- and anti-angiogenic drugs. This technique is performed either in-ovo or shell-less ex-ovo [59]. The ex ovo CAM assay is a method of assessing the anti-angiogenic potential of various test compound with minimal invasion. In present study, the chicken embryo cultured in a plastic cup (ex-ovo) provided a better access to the test site, allowed repeated treatments and multiple test [60]. The suitability of plastic cups based ex ovo assay for our study was also evident by the higher efficiency and viability of cultured chick embryos due to easy ventilation through the pores on transparent wraps or parafilm on plastic cups [61]. In addition, three to five days
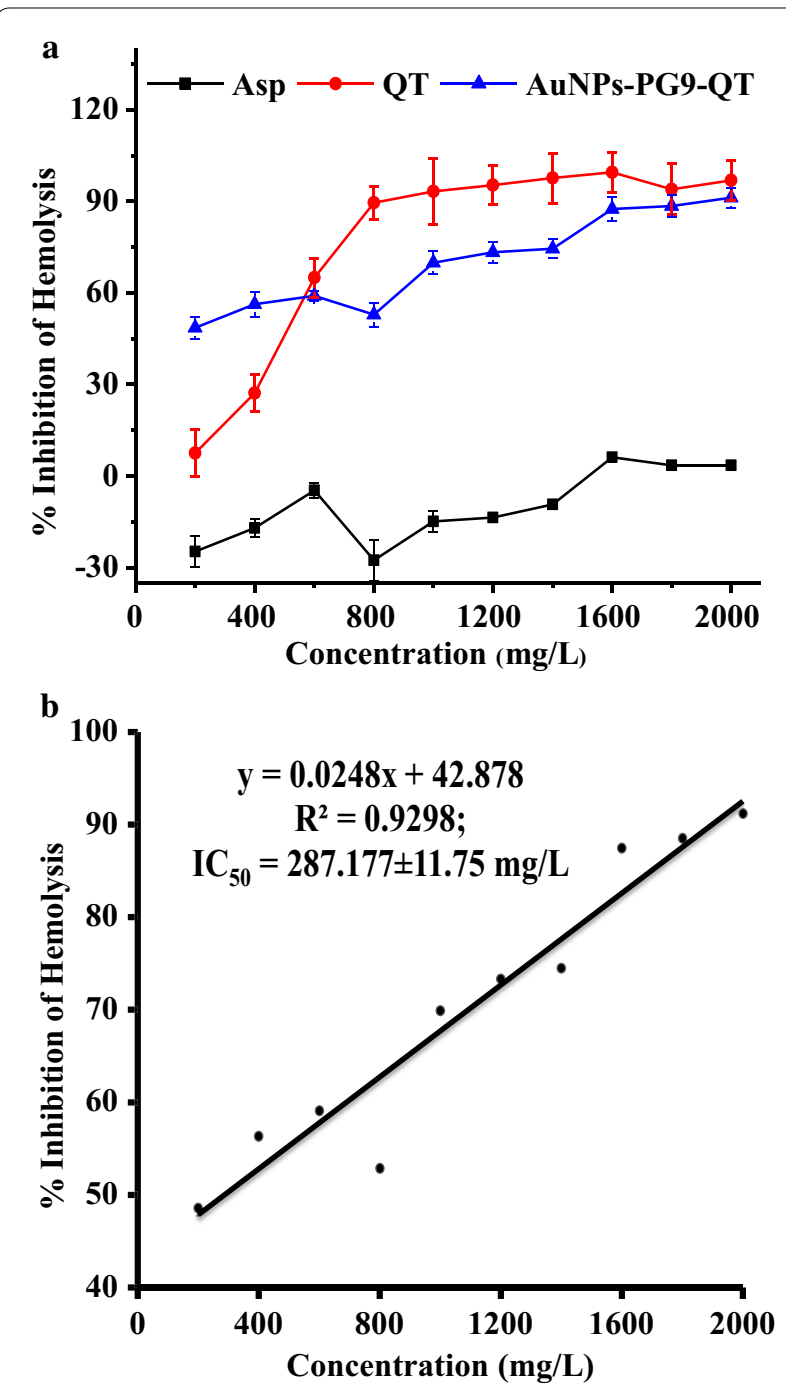

Fig. 6 Anti-inflammatory activity of polyethylene glycol 9000 capped and Quercetin functionalized AuNPs. a Effect of concentration of Aspirin, Quercetin and polyethylene glycol 9000 capped and Quercetin functionalized AuNPs on the \% inhibition of hemolysis and b a standard graph with line of regression showing direct correlation between concentrations of polyethylene glycol 9000 capped and Quercetin functionalized AuNPs and \% inhibition of hemolysis

were usually sufficient for this assay. In the present study, untreated hen CAMs (Fig. 7) showed normal vascular architecture with prominently branched-out, well-developed blood vessels indicating no major effect whereas, the treatment of CAMs with AuNPs-PG9-CPT in the range of $15-30 \mathrm{mg} / \mathrm{mL}$ resulted in marginal increase in inhibition of the blood vessel branching. The complete inhibition of blood vessel formation was observed in CAM treated with 30 and $40 \mathrm{mg} / \mathrm{mL}$ AuNPs-PG9-CPT. After $24 \mathrm{~h}$ of loading of 30 and $40 \mathrm{mg} / \mathrm{mL}$ AuNPs-PG9$\mathrm{CPT}$, the blood vessels below the disc were observed 


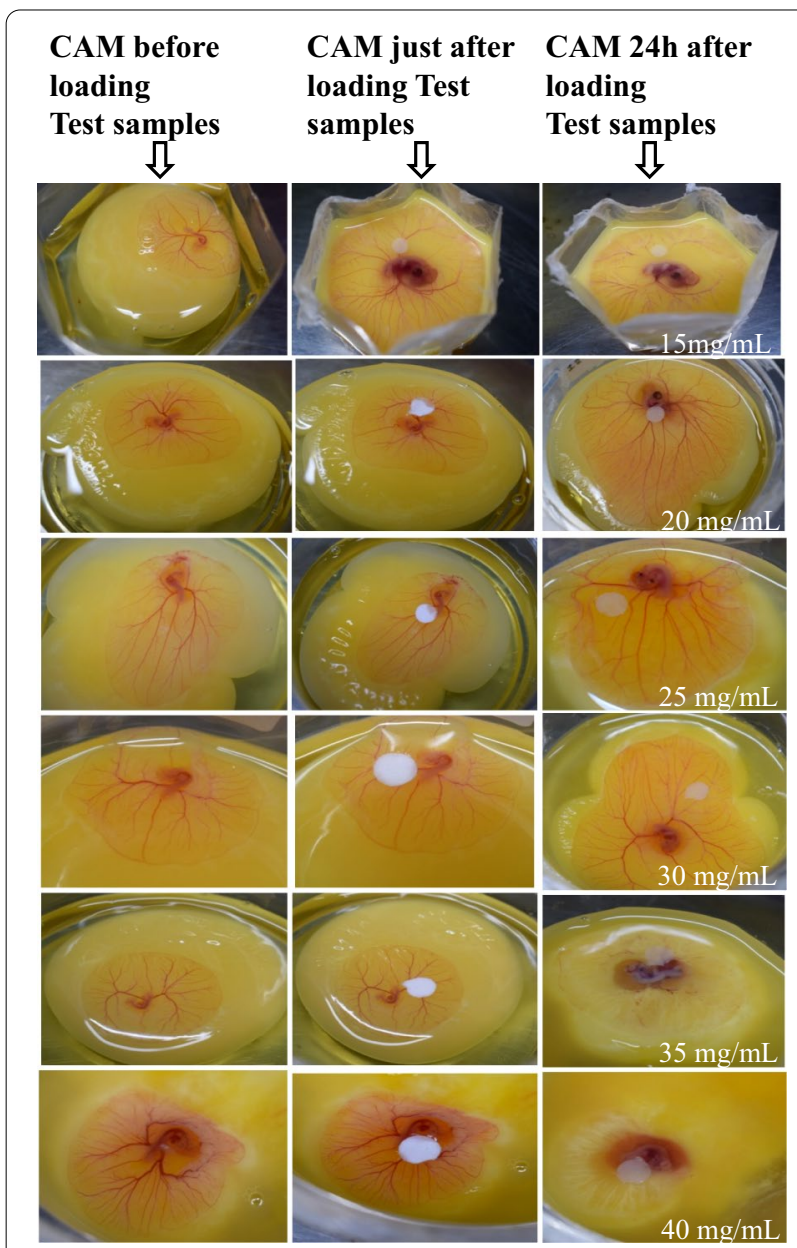

Fig. 7 Photographs of CAM showing the effect of polyethylene glycol 9000 capped and camptothecin functionalized AuNPs in the range of $15-40 \mathrm{mg} / \mathrm{mL}$ to disappear demonstrating excellent anti-angiogenic activity.

\section{Conclusion}

In conclusion, ASCE was successfully employed for optimized, rapid and cost-effective biogenic synthesis of AuNPs. Eight minutes of reaction, incubation at $90{ }^{\circ} \mathrm{C}$, reaction $\mathrm{pH}$ of $6,4 \mathrm{mM}$ of $\mathrm{HAuCl}_{4}$ and $4: 1$ ratio of extract to precursor were the optimum parameter for synthesis of crystalline, spherical AuNPs. The surface capping of AuNPs with PG9 was essential prior to functionalization with QT and CPT. The surface capped AuNPs were best functionalized at $70{ }^{\circ} \mathrm{C}$ with QT in $1 \mathrm{~h}$ reaction at pH 7 containing 1200 ppm of QT, and AuNPs-PG9:QT ratio of 1:4; whereas, $\mathrm{CPT}$ was best functionalized at $\mathrm{RT}$ in $1 \mathrm{~h}$ reaction with parameters such as $\mathrm{pH} 12$, AuNPsPG9:CPT ratio of $1: 1$ and $0.5 \mathrm{mM}$ of CPT. PG9 capped and QT functionalized AuNPs showed significant anti-cancer activity and ability to stabilize the RBCs membrane by inhibiting hemolysis whereas, PG9 capped and CPT functionalized AuNPs inhibited the CAM vascularization demonstrating excellent anti-angiogenic activity. Thus, the present study demonstrates an excellent application of drug functionalized biogenic AuNPs to develop nano-systems which can be used for biomedical applications.

\section{Materials and methods Materials}

All the chemicals and reagents used in the present study were of analytical (98\% pure) grade and used without any further purification. Mature, fully grown, dry Almond (Prunus amygdalus Syn. Prunus dulcis) nuts were purchased from the local market of Kolhapur, India. Gold (III) chloride trihydrate $\left(\mathrm{HAuCl}_{4} \cdot 3 \mathrm{H}_{2} \mathrm{O}\right)$, sodium hydroxide $(\mathrm{NaOH})$, dextrose $\left(\mathrm{C}_{6} \mathrm{H}_{12} \mathrm{O}_{6}\right)$, sodium citrate $\left(\mathrm{Na}_{3} \mathrm{C}_{6} \mathrm{H}_{5} \mathrm{O}_{7}\right)$, citric acid $\left(\mathrm{C}_{6} \mathrm{H}_{8} \mathrm{O}_{7}\right)$, sodium chloride $(\mathrm{NaCl})$,dimethyl sulfoxide (DMSO, 99\%), cell culture media-RPMI1640 supplemented with $10 \%$ foetal bovine serum (FBS), 1X Dulbecco's phosphate buffered saline (DPBS), 0.25\% Trypsin-EDTA solution and MTT reagent were purchased from Sigma-Aldrich (Sigma Aldrich, Mumbai, India). Quercetin was procured from Sigma-Aldrich (Sigma Aldrich, Mumbai, India) whereas; polyethylene glycol 6000 and 9000 (PG6 and PG9, respectively) and polyvinyl pyrrolidone K25 (PVP K25) were purchased from SRL (Sisco Research Laboratories Pvt. Ltd. Mumbai, India). Hydrochloric acid (HCL, 35.4\%) and absolute ethanol were purchased from Thomas Baker (Thomas Baker Chemicals, Pvt. Ltd. Mumbai, India). All the chemicals and reagents were prepared in sterile double distilled water (SDDW) unless otherwise mentioned.

\section{Preparation of almond seed coat extract}

In this study, leathery, brown seed coat of almond nuts was used to obtain phytochemicals rich extract for biogenic synthesis of AuNPs. Briefly, $200 \mathrm{~g}$ of almond nuts were thoroughly washed to remove surface impurities and soaked in SDDW at room temperature (RT) for $24 \mathrm{~h}$. Next day, almonds were observed for swelling and brown skins were hand peeled, washed for 3-4 times with SDDW, and then air dried at RT for $24 \mathrm{~h}$. After complete drying, skins were ground to fine powder using mixer grinder (Bajaj Electricals Limited, Mumbai, India). Thereafter, $8 \mathrm{~g}$ of powder was suspended in $150 \mathrm{~mL}$ of SDDW, stirred at $60{ }^{\circ} \mathrm{C}$ for $1 \mathrm{~h}$ in water bath. After incubation, the extract was cooled and filtered through muslin cloth to remove the particulate matter. The filtrate was finally centrifuged at 15,000 rpm for $20 \mathrm{~min}$ to obtained clear, light yellow ASCE which was then stored at $4-8{ }^{\circ} \mathrm{C}$ until further use. 


\section{Optimization of biogenic synthesis of AuNPs}

Initially, to study the ASCE's potential to synthesize AuNPs, $1 \mathrm{~mL}$ of $4 \mathrm{mM} \mathrm{HAuCl}$ precursor solution was $^{2}$ mixed with $3 \mathrm{~mL}$ of ASCE, incubated at $90{ }^{\circ} \mathrm{C}$ for $10 \mathrm{~min}$ with continuous stirring, monitored for the change in color from light yellow to dark ruby red as indication of AuNPs synthesis. In addition, AuNPs synthesis was also confirmed by recording the UV-Vis. spectra in the range of 250-800 $\mathrm{nm}$ on Biospectrophotometer (Eppendorf, Hamburg, Germany). Further to optimize the ASCE mediated synthesis; effects of variations in different synthesis parameters on AuNPs synthesis were studied. To accomplish this, reaction parameters such as incubation time, temperature, $\mathrm{pH}$, ratio of ASCE:precursor and precursor $\left(\mathrm{HAuCl}_{4}\right)$ concentration were varied in the ranges of 0-10 min, $50-90{ }^{\circ} \mathrm{C}, 2-10,1: 1-5: 1$ and $0.25-1.5 \mathrm{mM}$, respectively. For all these optimization experiments also, reactions were monitored initially bythe change of color and then by recording UV-Vis spectra in the range of 250-800 $\mathrm{nm}$. After optimization, large scale synthesis was carried out using optimized conditions; the colloidal solution of AuNPs was then centrifuged at 25,000 rpm for $15 \mathrm{~min}$ to collect AuNPs pellet. The pellet was washed 3-4 times, dried at $60{ }^{\circ} \mathrm{C}$ in hot air oven for $12 \mathrm{~h}$, and characterized using different spectroscopy and imaging techniques.

\section{Characterization of biogenic AuNPs}

In this study, optimally synthesized AuNPs were confirmed by recording UV-Vis. spectra whereas the elemental composition was confirmed by energy dispersive X-ray spectroscopy (EDS) using AztecLive EDS analysis software (Oxford Instruments, UK). Further, Fourier transform infrared spectroscopy (FTIR) analyses of dried ASCE and AuNPs were carried out to study the functional groups of different biomolecules of ASCE that could have functioned as reducing and capping agents. To this end, $2 \mathrm{mg}$ each of AuNPs and ASCE were mixed with $200 \mathrm{mg}$ of potassium bromide $(\mathrm{KBr})$ and pellet were prepared by applying pressure of 6-7 tons to obtain pellets of $1-1.5 \mathrm{~mm}$ thickness. FTIR spectra were then recorded in transmittance mode over the range of $400-4000 \mathrm{~cm}^{-1}$ on FTIR spectrometer (Bruker alpha Shimadzu, Japan). The X-ray diffraction (XRD) pattern analysis was employed to study the crystalline nature of AuNPs by recording XRD pattern on Bruker's AXS analytical instruments (Bruker Pvt. Ltd., Germany). In addition, size, morphologyand selected area electron diffraction pattern (SAED) of AuNPs were imaged using high resolution transmission electron microscope (HR-TEM) operating at an acceleration voltage of $200 \mathrm{kV}$ (Jeol Ltd., Tokyo, Japan). The average size of AuNPs was estimated by dynamic light scattering (DLS) and zeta potential (surface charge) measurement was done by PALS zeta potential analyser var. 5.76 (Brookhaven Instrument Corp. Holtsville, New York, USA).

\section{Surface capping and functionalization of AuNPs with quercetin and camptothecin}

In order to facilitate and enhance the functionalization of AuNPs with QT and CPT, initially three different polymers namely PG6, PG9 and PVP K25 were screened and evaluated for their potential utility in surface capping prior to functionalization. Briefly, freshly prepared $1 \mathrm{~mL}$ of $0.1 \mathrm{M}$ of aqueous solutions of PG6, PG9 and PVP K25 were added separately to the $1 \mathrm{~mL}$ of neat AuNPs. The solutions were stirred at RT for $24 \mathrm{~h}$ to allow the complete exchange of AuNPs surface molecules with capping agents. To confirm the pegylation and capping of AuNPs with PVP K25, the preparations were analyzed by recording UV-Vis. spectra in the range of $250-800 \mathrm{~nm}$. During capping reaction, red shift of the SPR band resulting from increased refractive index surrounding the AuNPs due to the modification of capping agent was monitored [44]. The preparations showing maximum absorbance with redshift in the SPR were selected as successfully capped and used for functionalization with QT and CPT.

To functionalize the capped AuNPs (CAuNPs) with QT (CAuNPs-QT), an initial reaction was carried out by mixing CAuNPs with QT (1000 ppm) in 1:3 ratio, pH 6 and stirred at $50{ }^{\circ} \mathrm{C}$ for $2 \mathrm{~h}$. In order to optimize the functionalization reaction parameters and study how the variations in different parameters such as incubation time, $\mathrm{pH}$, temperature, concentration of QT and CAuNPs:QT ratio influence the functionalization, they were varied in the ranges of $0-120 \mathrm{~min}, 4-7,30-70{ }^{\circ} \mathrm{C}, 1: 1-1: 4$ and 400-1600 ppm, respectively. In case of functionalization of CAuNPs with CPT (CAuNPs-CPT), initially $0.5 \mathrm{mM}$ $\mathrm{CPT}$ in 5\% DMSO was mixed with CAuNPs in 1:1 ratio, $\mathrm{pH} 7$ and stirred at RT for $1 \mathrm{~h}$. Later, the functionalization conditions were optimized by studying the effects of variations in reaction time, concentration of $\mathrm{CPT}$, ratio and $\mathrm{pH}$ etc. in the ranges of $1-6 \mathrm{~h}, 0.2-0.5 \mathrm{mM}$, ratio $1: 1$ and $\mathrm{pH} 2-12$ respectively. To confirm the functionalization, the reactions were initially analyzed by recording UV-Vis. spectra throughout the experiments. After optimization, large scale functionalization were done using optimized parameters, CAuNPs-QT and CAuNPs-CPT were then centrifuged at 20,000 rpm for $15 \mathrm{~min}$ and pellets were washed with SDDW to remove unbound QT, $\mathrm{CPT}$ and organic impurities. Further, CAuNPs-QT and CAuNPs-CPT were dried $40^{\circ} \mathrm{C}$, characterized using EDS 
and FTIR spectroscopy and stored at RT until further use.

\section{Anti-cancer activity of quercetin functionalized AuNPs}

The anti-cancer activity of AuNPs-PG9-QT was tested against Human Breast Cancer Cell line MCF-7 using MTT assay. Briefly, MCF-7 cells cultured in T-25 flasks were trypsinized and aspirated into a $5 \mathrm{~mL}$ centrifuge tube. Cell pellet was obtained by centrifugation at $300 \times \mathrm{g}$, cell count was adjusted using DMEM HG medium such that $200 \mu \mathrm{L}$ of suspension contained approximately 10,000 cells. To each well of the 96 well micro-titre plate, $200 \mu \mathrm{L}$ of the cell suspension was added and the plate was incubated at $37{ }^{\circ} \mathrm{C}$ and $5 \% \mathrm{CO}_{2}$ atmosphere for $24 \mathrm{~h}$. After $24 \mathrm{~h}$, the spent medium was aspirated, $200 \mu \mathrm{L}$ of test samples such as QT, AuNPs and AuNPs-PG9-QT to the final concentrations in the range of $25-800 \mu \mathrm{g} /$ $\mathrm{mL}$ were added to the respective wells. The standard drug Cisplatin was also included in the assay. The plate was then incubated at $37{ }^{\circ} \mathrm{C}$ and $5 \% \mathrm{CO}_{2}$ atmosphere for $24 \mathrm{~h}$. Thereafter, the plate was removed from the incubator, the drug containing media was aspirated, $200 \mu \mathrm{L}$ of medium containing 10\% MTT reagent was added to each well to get a final concentration of $0.5 \mathrm{mg} / \mathrm{mL}$ and then the plate was incubated at $37{ }^{\circ} \mathrm{C}, 5 \% \mathrm{CO}_{2}$ atmosphere for $3 \mathrm{~h}$. Next, the culture medium was removed completely without disturbing the crystals formed, $100 \mu \mathrm{L}$ of solubilization solution (DMSO) was added and the plate was gently shaken in a gyratory shaker to solubilize the formed formazan. The absorbances were measured using a microplate reader at a wavelength of $570 \mathrm{~nm}$ and also at $630 \mathrm{~nm}$. The percentage growth inhibition was calculated after subtracting the background and the blank, and concentration of test sample needed to inhibit cell growth by $50 \%\left(\mathrm{IC}_{50}\right)$ was estimated from the dose-response curve for the cell line. The percentage (\%) of cell viability was calculated using following formula:

$$
\% \text { cell viability }=\frac{\text { Absorbance of sample }}{\text { Absorbance of control }} \times 100 \%
$$

\section{Anti-inflammatory activity of quercetin functionalized PG9 capped AuNPs}

The anti-inflammatory activity of AuNPs-PG9-QT was assessed by employing the membrane stabilization test based on inhibition of heat induced hemolysis of RBCs. To accomplish this, fresh human blood was collected at Health Centre Hospital of Shivaji University, Kolhapur by following the standard medical practices and mixed with equal volume of sterilized Alsever's solution ( $2 \%$ dextrose, $0.8 \%$ sodium citrate, $0.05 \%$ citric acid and $0.42 \%$ sodium chloride). The mixture was then centrifuged at $3000 \mathrm{rpm}$ for $10 \mathrm{~min}$, the packed cells were washed thrice with saline $(0.85 \%, \mathrm{pH} 7.2)$, and the obtained blood volume was measured and reconstituted as $10 \%(\mathrm{v} / \mathrm{v})$ suspension with saline. For the test of anti-inflammatory activity, the heat induced hemolysis reactions consisted of $1 \mathrm{~mL}$ of $10 \%$ RBCs suspension and $1 \mathrm{~mL}$ of AuNPs-PG9-QT to the final concentrations in the range of $200-2000 \mathrm{mg} / \mathrm{L}$. Saline solution was added instead of test sample to the blank test tube while Aspirin (Asp) and QT were used as standard and control drugs, respectively. All the reactions were incubated in a water bath at $56{ }^{\circ} \mathrm{C}$ for $30 \mathrm{~min}$., cooled under running tap water and centrifuged at $2500 \mathrm{rpm}$ for $5 \mathrm{~min}$. The absorbance of supernatant was recorded at $560 \mathrm{~nm}$. The experiments were performed in triplicates for all test samples. The \% inhibition of hemolysis was calculated by using the formula given below:

\%inhibition

$$
=\frac{\text { Absorbance of control }- \text { Absorbance of sample }}{\text { Absorbance of control }} \times 100 \%
$$

\section{Anti-angiogenic activity of camptothecin functionalized AuNPs}

For the test of CAM based anti-angiogenic activity of AuNPs-PG9-CPT, zero-day old eggs were obtained from the Egg hatchery center, Kolhapur, India. All the handling and experimental steps were performed in laminar air flow (LAF) hood and all tools were cleaned and surface sterilized with $70 \%$ ethanol. Prior to the CAM assay, the outer surfaces of the eggs were cleaned with SDDW, placed vertically into egg incubator and incubated at $37{ }^{\circ} \mathrm{C}$ and $70 \%$ humidity for $72 \mathrm{~h}$. After $72 \mathrm{~h}$, eggs were kept horizontally for one hour without rotation to bring the CAM on upper side of the egg and facilitate the transfer of an intact embryo to the plastic cups for ex-ovo culture. Thereafter, the eggs were removed from the incubator, transferred to LAF hood, cracked open gently using metal bar and the contents were carefully transferred to the surface sterilized plastic cups. Further, the plastic cups were covered with parafilm, the ex-ovo cultures were returned to the egg incubator with minimal disturbances and dead embryos were removed to minimize infection on the CAM and increase their survival rates. Next day, the numbers of blood vessels of the embryos were counted prior to the application of the filter paper discs loaded with test samples. For efficient observation of the effects of test samples on the angiogenesis, discs were placed directly using a sterile forceps over the blood vessels on the growing CAM where two major blood vessels bifurcated. To this end, the circular discs were loaded 
with AuNPs-PG9-CPT in the range of $15-40 \mathrm{mg} / \mathrm{mL}$ along with standard drug CPT $(0.5 \mathrm{mM})$. Subsequently, the ex-ovo cultures were returned to the egg incubator, and the final evaluations were carried out on day 5 . The number of blood vessels was counted at the site of sample application, photographed and the comparison was done before and after treatment.

\section{Abbreviations}

ASCE: Almond seed coat extract; SDDW: Sterile Double distilled water; NPs: Nanoparticles; MNPs: Metal nanoparticles; NMs: Nanomaterials; RT: Room temperature; AuNPs: Gold nanoparticles; SPR: Surface plasmon resonance; Au: Elemental Gold; PG9: Polyethylene glycol 9000; PG6: Polyethylene glycol 6000; PVP K25: Polyvinyl pyrrolidoneK25; AuNPs-PG6: PG6 capped Gold nanoparticles; AuNPs-PG9: PG9 capped Gold nanoparticles; AuNPs-PVP K25: PVP K25 capped Gold nanoparticles; CAuNPs: Capped AuNPs; QT: Quercetin; AuNPs-PG9-QT: Quercetin functionalized PG9 capped Gold nanoparticles; CAuNPs-QT: QT functionalize capped AuNPs; CPT: Camptothecin; CAuNPs-CPT: CPT functionalize capped AuNPs; AuNPs-PG9-CPT: Camptothecin functionalized PEG 9000 capped Gold nanoparticles; MTT: 3-(4,5-Dimethylthiahiazol2-yl)-2,5-diphenyl tetrazolium; Asp: Aspirin; RBCs: Red blood cells; PBS: Phosphate Buffered Saline; CAM: Chorioallantoic membrane assay.

\section{Supplementary Information}

The online version contains supplementary material available at https://doi. org/10.1186/s12951-021-00836-1.

Additional file 1: Figure S1. UV-Vis spectra surface capping reactions of AuNPs with different capping agents. Figure S2. UV-Vis absorption spectra showing effect of different (a) AuNPs-PG9-QT synthesis, (b) Incubation times, (c) pH, (d) ratio of AuNPs-PG9:QT, (e) incubation temperature and (f) concentration of QT on AuNPs-PG9-QT synthesis. Figure S3. UV-Vis absorption spectra showing effect of different physico-chemical parameters (a) concentration of CPT, (b) incubation times, (c) pH on AuNPs-PG9CPT synthesis. Table S1. Fourier transform-infrared spectroscopy based analysis of ASCE and AuNPs for the study of vibrational stretchings and corresponding functional groups. Table S2. Fourier transform-infrared spectroscopy based analysis of QT and AuNPs-PG9-QT for the study of vibrational stretchings and corresponding functional groups (DOCX 1802 $\mathrm{KB})$

\section{Acknowledgement}

The authors gratefully acknowledge use of research infrastructure and facility of DBT-BUILDER programme sanctioned to Shivaji University, Kolhapur. Also, STIC Cochin is acknowledge for providing HR-TEM facility.

\section{Authors' contributions}

PS has contributed in ASCE preparation and characterization, designing and performance of the experiments, graphics preparation, analyzed \& validated the data of synthesized, optimized, capped \& functionalized (AuNPs-PG9-QT), characterized AuNPs, and has organized the figure data, wrote \& revised the manuscript. RP contributed to the data analysis of anti-cancer, anti-inflammatory \& anti-angiogenic activity, writing and revision of the manuscript has done with the help of DH and SG. RP and DH contributed equally to this work. DH performed surface capping and functionalization of AuNPs with QT, contributed to the sample preparation and data analysis of anti-cancer study, methodology \& analysis of anti-inflammatory study. SG performed the experiment of capping and functionalization of AuNPs with CPT, anti-angiogenic activity and data analysis. AS contributed to methodology, review \& editing of the manuscript. KP contributed to the conception of the study, supervision, validation of data and has reviewed \& edited the manuscript. The corresponding author had full access to all the data in the study and had final responsibility for the decision to submit for publication. All authors read and approved the final manuscript.
Funding

Not Applicable.

\section{Availability of data and materials}

All data generated or analysed during this study are included in this published article and its supplementary information files.

\section{Declarations}

Ethics approval and consent to participate

Not Applicable.

Consent for publication

Not Applicable.

\section{Competing interests}

The authors declare that they have no competing interests.

\section{Author details}

${ }^{1}$ School of Nanoscience and Biotechnology, Shivaji University, Kolhapur, Maharashtra, India. ${ }^{2}$ Department of Pharmaceutical Technology, Faculty of Pharmacy, University of Coimbra, Coimbra, Portugal. ${ }^{3}$ REQUIMTE/LAQV, Group of Pharmaceutical Technology, Faculty of Pharmacy, University of Coimbra, Coimbra, Portugal.

Received: 27 September 2020 Accepted: 16 March 2021

Published online: 25 March 2021

\section{References}

1. Ma X, Yu H. Global burden of cancer. Yale J Biol Med. 2006;79:85-94.

2. Bray F, Ferlay J, Soerjomataram I, Siegel RL, Torre LA, Jemal A. Global cancer statistics 2018: GLOBOCAN estimates of incidence and mortality worldwide for 36 cancers in 185 countries. CA Cancer J Clin. 2018;68:394-424

3. Chan K, Morris GJ. Chemoprevention of breast cancer for women at high risk. Semin Oncol. 2006:33:642-6.

4. Tinoco G, Warsch S, Glück S, Avancha K, Montero AJ. Treating breast cancer in the 21st century: emerging biological therapies. J Cancer. 2013:4:117-32

5. Patra JK, Das G, Fraceto LF, Campos EVR, Rodriguez-Torres MDP, AcostaTorres LS, et al. Nano based drug delivery systems: Recent developments and future prospects 10 Technology 1007 Nanotechnology 03 Chemical Sciences 0306 Physical Chemistry (incl Structural) 03 Chemical Sciences 0303 Macromolecular and Materials Chemistry 11 Medical and He. J Nanobiotechnol BioMed Central. 2018;16:1-33. https://doi.org/10.1186/ s12951-018-0392-8.

6. Grace Intasa-ard S, Birault A. Nanoparticles characterization using the CAM assay. Enzymes. 1st ed. Amsterdam: Elsevier Inc; 2019.

7. Whitesides GM. Nanoscience, nanotechnology, and chemistry. Small. 2005;1:172-9.

8. Cobley CM, Chen J, Chul Cho E, Wang LV, Xia Y. Gold nanostructures: a class of multifunctional materials for biomedical applications. Chem Soc Rev. 2011:40:44-56.

9. Dreaden EC, Mackey MA, Huang X, Kang B, El Sayed MA. Beating cancer in multiple ways using nano gold. Chem Soc Rev. 2011:40:3391-404.

10. Sengani M, Grumezescu AM, Rajeswari VD. Recent trends and methodologies in gold nanoparticle synthesis-a prospective review on drug delivery aspect. OpenNano. 2017;2:37-46. https://doi.org/10.1016/j. onano.2017.07.001.

11. Tang L, Yang X, Yin Q, Cai K, Wang H, Chaudhury I, et al. Investigating the optimal size of anticancer nanomedicine. Proc Natl Acad Sci USA 2014;111:15344-9.

12. El Domany EB, Essam TM, Ahmed AE, Farghali AA. Biosynthesis physicochemical optimization of gold nanoparticles as anti-cancer and synergetic antimicrobial activity using Pleurotus ostreatus fungus. J Appl Pharm Sci. 2018:8:119-28. 
13. Darweesh RS, Ayoub NM, Nazzal S. Gold nanoparticles and angiogenesis: Molecular mechanisms and biomedical applications. Int J Nanomed. 2019;14:7643-63.

14. Yguerabide J, Yguerabide EE. Light-scattering submicroscopic particles as highly fluorescent analogs and their use as tracer labels in clinical and biological applications II. Exp Charact Anal Biochem. 1998;262:157-76.

15. Madhusudhan A, Reddy GB, Venkatesham M, Veerabhadram G, Kumar $A D$, Natarajan S, et al. Efficient ph dependent drug delivery to target cancer cells by gold nanoparticles capped with carboxymethyl chitosan. Int J Mol Sci. 2014;15:8216-34.

16. Lee KX, Shameli K, Yew YP, Teow SY, Jahangirian H, Rafiee-Moghaddam R, et al. Recent developments in the facile bio-synthesis of gold nanoparticles (AuNPs) and their biomedical applications. Int J Nanomed. 2020;15:275-300.

17. Narayanan KB, Sakthivel N. Biological synthesis of metal nanoparticles by microbes. Adv Colloid Interface Sci. 2010;156:1-13. https://doi.org/10. 1016/j.cis.2010.02.001

18. Rajakumar G, Gomathi T, Rahuman AA, Thiruvengadam M, Mydhili G, Kim $\mathrm{SH}$, et al. Biosynthesis and biomedical applications of gold nanoparticles using eclipta prostrata leaf extract. Appl Sci. 2016;6(8):222. https://doi. org/10.3390/app6080222.

19. Husseiny MI, El-Aziz MA, Badr Y, Mahmoud MA. Biosynthesis of gold nanoparticles using Pseudomonas aeruginosa. Spectrochim Acta Part A Mol Biomol Spectrosc. 2007:67:1003-6.

20. Pimprikar PS, Joshi SS, Kumar AR, Zinjarde SS, Kulkarni SK. Influence of biomass and gold salt concentration on nanoparticle synthesis by the tropical marine yeast Yarrowia lipolytica NCIM 3589. Colloids Surfaces B Biointerfaces. 2009;74:309-16.

21. Princy KF, Gopinath A. Optimization of physicochemical parameters in the biofabrication of gold nanoparticles using marine macroalgae Padina tetrastromatica and its catalytic efficacy in the degradation of organic dyes. J Nanostructure Chem. 2018;8:333-42. https://doi.org/10.1007/ s40097-018-0277-2.

22. El Kurdi R, Patra D. The role of $\mathrm{OH}$ - in the formation of highly selective gold nanowires at extreme $\mathrm{pH}$ : Multi-fold enhancement in the rate of the catalytic reduction reaction by gold nanowires. Phys Chem Chem Phys. 2017;19:5077-90.

23. Al Shehab S, El Kurdi R, Patra D. Curcumin mediated PEG thiol acid conjugated gold nanoparticles for the determination of melamine. Microchem J. 2020;153:104382. https://doi.org/10.1016/j.microc.2019.104382.

24. Khalighi Sheshdeh R, Khosravi Nikou MR, Badii K, Mohammadzadeh S. Evaluation of adsorption kinetics and equilibrium for the removal of benzene by modified diatomite. Chem Eng Technol. 2013;36:1713-20.

25. Singh P, Kim YJ, Zhang D, Yang DC. Biological synthesis of nanoparticles from plants and microorganisms. Trends Biotechnol. 2016;34:588-99. https://doi.org/10.1016/j.tibtech.2016.02.006.

26. Shah M, Fawcett D, Sharma S, Tripathy SK, Poinern GEJ. Green synthesis of metallic nanoparticles via biological entities. Materials (Basel). 2015;8(11):7278-308.

27. Shankar SS, Rai A, Ahmad A, Sastry M. Rapid synthesis of Au, Ag, and bimetallic Au core-Ag shell nanoparticles using Neem (Azadirachta indica) leaf broth. J Colloid Interface Sci. 2004;275:496-502.

28. Jha AK, Prasad K, Prasad K, Kulkarni AR. Plant system: nature's nanofactory. Colloids Surfaces B Biointerfaces. 2009;73:219-23.

29. Philip D. Rapid green synthesis of spherical gold nanoparticles using Mangifera indica leaf. Spectrochim Acta Part A Mol Biomol Spectrosc. 2010;77:807-10. https://doi.org/10.1016/j.saa.2010.08.008.

30. Song JY, Jang HK, Kim BS. Biological synthesis of gold nanoparticles using Magnolia kobus and Diopyros kaki leaf extracts. Process Biochem. 2009:44:1133-8.

31. Ramamurthy $\mathrm{CH}$, Padma M, Mariya Samadanam ID, Mareeswaran R, Suyavaran A, Kumar MS, et al. The extra cellular synthesis of gold and silver nanoparticles and their free radical scavenging and antibacterial properties. Colloids Surfaces B Biointerfaces. 2013;102:808-15. https://doi. org/10.1016/j.colsurfb.2012.09.025.

32. Desai MP, Sangaokar GM, Pawar KD. Kokum fruit mediated biogenic gold nanoparticles with photoluminescent, photocatalytic and antioxidant activities. Process Biochem. 2018;70:188-97. https://doi.org/10.1016/j. procbio.2018.03.027.

33. Katas H, Moden NZ, Lim CS, Celesistinus T, Chan JY, Ganasan P, et al. Biosynthesis and potential applications of silver and gold nanoparticles and their chitosan-based nanocomposites in nanomedicine. J Nanotechnol. 2018;2018:4290705. https://doi.org/10.1155/2018/4290705.

34. Kumari M, Mishra A, Pandey S, Singh SP, Chaudhry V, Mudiam MKR, et al Physico-chemical condition optimization during biosynthesis lead to development of improved and catalytically efficient gold nano particles. Sci Rep. 2016;6:1-14. https://doi.org/10.1038/srep27575.

35. Singh C, Baboota RK, Naik PK, Singh H. Biocompatible synthesis of silver and gold nanoparticles using leaf extract of Dalbergia sissoo. Adv Mater Lett. 2012;3:279-85.

36. Ndeh NT, Maensiri S, Maensiri D. The effect of green synthesized gold nanoparticles on rice germination and roots. Adv Nat Sci Nanosci Nanotechnol. 2017;8:035008.

37. Dwivedi AD, Gopal K. Biosynthesis of silver and gold nanoparticles using Chenopodium album leaf extract. Colloids Surfaces A Physicochem Eng Asp. 2010;369:27-33. https://doi.org/10.1016/j.colsurfa.2010.07.020.

38. Khalil MMH, Ismail EH, El-Magdoub F. Biosynthesis of Au nanoparticles using olive leaf extract. 1st nano updates. Arab J Chem. 2012;5:431-7. https://doi.org/10.1016/j.arabjc.2010.11.011.

39. Wei X, Qi L, Tan J, Liu R, Wang F. A colorimetric sensor for determination of cysteine by carboxymethyl cellulose-functionalized gold nanoparticles. Anal Chim Acta. 2010;671:80-4. https://doi.org/10.1016/j.aca.2010.05.006.

40. Babu PJ, Saranya S, Sharma P, Tamuli R, Bora U. Gold nanoparticles: Sonocatalytic synthesis using ethanolic extract of Andrographis paniculata and functionalization with polycaprolactone-gelatin composites. Front Mater Sci. 2012;6:236-49.

41. Arunachalam KD, Annamalai SK, Hari S. One-step green synthesis and characterization of leaf extract-mediated biocompatible silver and gold nanoparticles from Memecylon umbellatum. Int J Nanomedicine. 2013:8:1307-15.

42. Geetha R, Ashokkumar T, Tamilselvan S, Govindaraju K, Sadiq M, Singaravelu $\mathrm{G}$. Green synthesis of gold nanoparticles and their anticancer activity. Cancer Nanotechnol. 2013;4:91-8.

43. Oh KS, Kim RS, Lee J, Kim D, Cho SH, Yuk SH. Gold/chitosan/pluronic composite nanoparticles for drug delivery. J Appl Polym Sci. 2008;108:3239-44.

44. Nam S, Parikh DV, Condon BD, Zhao Q, Yoshioka-Tarver M. Importance of poly(ethylene glycol) conformation for the synthesis of silver nanoparticles in aqueous solution. J Nanoparticle Res. 2011;13:3755-64.

45. Stiufiuc R, lacovita C, Nicoara R, Stiufiuc G, Florea A, Achim M, et al. Onestep synthesis of PEGylated gold nanoparticles with tunable surface charge. J Nanomater. 2013;2013:1-7. https://doi.org/10.1155/2013/ 146031.

46. Miguel-García I, Berenguer-Murcia Á, García T, Cazorla-Amorós D. Effect of the aging time of PVP coated palladium nanoparticles colloidal suspensions on their catalytic activity in the preferential oxidation of CO. Catal Today. 2012;187:2-9. https://doi.org/10.1016/j.cattod.2012.02.015.

47. Pal R, Panigrahi S, Bhattacharyya D, Chakraborti AS. Characterization of citrate capped gold nanoparticle-quercetin complex: Experimental and quantum chemical approach. J Mol Struct. 2013;1046:153-63. https://doi. org/10.1016/j.molstruc.2013.04.043.

48. He B, Tan JJ, Liew KY, Liu H. Synthesis of size controlled Ag nanoparticles. J Mol Catal A Chem. 2004;221:121-6.

49. Rawat KA, Kailasa SK. Visual detection of arginine, histidine and lysine using quercetin-functionalized gold nanoparticles. Microchim Acta. 2014;181:1917-29.

50. Cao Q, Zhao H, He Y, Li X, Zeng L, Ding N, et al. Hydrogen-bondinginduced colorimetric detection of melamine by nonaggregation-based Au-NPs as a probe. Biosens Bioelectron. 2010;25:2680-5. https://doi.org/ 10.1016/j.bios.2010.04.046.

51. Kurepa J, Nakabayashi R, Paunesku T, Suzuki M, Saito K, Woloschak GE, et al. Direct isolation of flavonoids from plants using ultra-small anatase TiO2 nanoparticles. Plant J. 2014;77:443-53.

52. Rameshthangam P, Chitra JP. Synergistic anticancer effect of green synthesized nickel nanoparticles and quercetin extracted from Ocimum sanctum leaf extract. J Mater Sci Technol. 2018;34:508-22. https://doi.org/ 10.1016/j.jmst.2017.01.004.

53. Hussein-Al-Ali SH, Arulselvan P, Fakurazi S, Hussein MZ, Dorniani D. Arginine-chitosan- and Arginine-polyethylene glycol-conjugated superparamagnetic nanoparticles: preparation, cytotoxicity and controlled-release. J Biomater Appl. 2014;29:186-98. 
54. Firoozeh N, Mahmoud O, Layasadat K, Mohammadreza A, Esrafil M, Ali K Effects of quercetin-loaded nanoparticles on MCF-7. Medicina (B Aires). 2019;55:1-15.

55. Sakat SS, Juvekar AR, Gambhire MN. In-vitro antioxidant and anti-inflammatory activity of methanol extract of Oxalis corniculata linn. Int J Pharm Pharm Sci. 2010;2:146-55.

56. Shinde UA, Kulkarni KR, Phadke AS, Nair AM, Mungantiwar AA, Dikshit VJ, et al. Mast cell stabilizing and lipoxygenase inhibitory activity of Cedrus deodara (roxb.) Loud. wood oil. Indian J Exp Biol. 1999;37:258-61.

57. Mukherjee S, Patra CR. Therapeutic application of anti-angiogenic nanomaterials in cancers. Nanoscale. 2016:8:12444-70.

58. Ribatti D. The chick embryo chorioallantoic membrane as a model tumor biology. Exp. Cell Res. 2014;328:314-24. https://doi.org/10.1016/j.yexcr. 2014.06.010.
59. Vargas A, Zeisser-Labouèbe M, Lange N, Gurny R, Delie F. The chick embryo and its chorioallantoic membrane (CAM) for the in vivo evaluation of drug delivery systems. Adv Drug Deliv Rev. 2007;59:1162-76.

60. Staton CA, Reed MWR, Brown NJ. A critical analysis of current in vitro and in vivo angiogenesis assays. Int J Exp Pathol. 2009;90:195-221.

61. Naik M, Brahma P, Dixit M. A cost-effective and efficient chick ex-ovo cam assay protocol to assess angiogenesis. Methods Protoc. 2018;1:1-9.

\section{Publisher's Note}

Springer Nature remains neutral with regard to jurisdictional claims in published maps and institutional affiliations.
Ready to submit your research? Choose BMC and benefit from:

- fast, convenient online submission

- thorough peer review by experienced researchers in your field

- rapid publication on acceptance

- support for research data, including large and complex data types

- gold Open Access which fosters wider collaboration and increased citations

- maximum visibility for your research: over $100 \mathrm{M}$ website views per year

At BMC, research is always in progress.

Learn more biomedcentral.com/submissions 